\title{
RhoGAP19D inhibits Cdc42 laterally to control epithelial cell shape and prevent invasion
}

Weronika Fic ${ }^{1}$, Rebecca Bastock ${ }^{1,4}$, Francesco Raimondi ${ }^{2,5}$, Erinn Los ${ }^{1}$, Yoshiko Inoue $^{3,6}$, Jennifer Gallop ${ }^{3}$, Robert B. Russell ${ }^{2}$ and Daniel St Johnston ${ }^{1,7}$

${ }^{1}$ The Gurdon Institute and the Department of Genetics, University of Cambridge, Tennis Court Road, Cambridge CB2 1QN, United Kingdom

${ }^{2}$ BioQuant and Biochemie Zentrum Heidelberg (BZH), Heidelberg University, Im Neuenheimer Feld 267, 69120 Heidelberg, Germany

${ }^{3}$ The Gurdon Institute and the Department of Biochemistry, University of Cambridge, Tennis Court Road, Cambridge CB2 1QN, United Kingdom

${ }^{4}$ Current address: Sheffield Diagnostic Genetics Service, Sheffield Children's NHS Trust, Western Bank, Sheffield, United Kingdom

${ }^{5}$ Current address: Bio@SNS, Scuola Normale Superiore, Pisa, Italy

${ }^{6}$ Current address: Research Organization of Information and Systems, Hulic Kamiyacho Building 2F, Toranomon 4-3-13, Minato-ku, Tokyo 105-0001

${ }^{7}$ Corresponding author: d.stjohnston@gurdon.cam.ac.uk 


\section{Summary}

Cdc42-GTP is required for apical domain formation in epithelial cells where it recruits and activates the Par-6/aPKC polarity complex, but how the activity of Cdc42 itself is restricted apically unclear. We used sequence analysis and 3D structure modelling to determine which Drosophila GTPase Activating Proteins (GAPs) are likely to interact with Cdc42 and identified RhoGAP19D as the sole Cdc42GAP required for polarity in the follicle cell epithelium. RhoGAP19D is recruited by $\alpha-$ catenin to lateral E-cadherin adhesion complexes, resulting in exclusion of active Cdc42 from the lateral domain. rhogap 19d mutants therefore lead to lateral Cdc42 activity, which expands the apical domain through increased Par-6/aPKC activity and stimulates lateral contractility through the myosin light chain kinase, Genghis khan (MRCK). This causes buckling of the epithelium and invasion into the adjacent tissue, a phenotype resembling that of pre-cancerous breast lesions. Thus, RhoGAP19D couples lateral Cadherin adhesion to the apical localisation of active Cdc42, thereby suppressing epithelial invasion.

\section{Introduction}

The form and function of epithelial cells depends on their polarisation into distinct apical, lateral and basal domains by conserved polarity factors (Rodriguez-Boulan and Macara, 2014; St Johnston and Ahringer, 2010). This polarity is then maintained by mutual antagonism between apical polarity factors like atypical protein kinase $\mathrm{C}$ (aPKC) and lateral factors, such as Lgl and Par-1. While many aspects of the polarity machinery are now well understood, it is still unclear how the apical domain is initiated and what role Cdc42 plays in this process.

Cdc42 was identified for its role in establishing polarity in budding yeast, where it targets cell growth to the bud tip by polarising the actin cytoskeleton and exocytosis towards a single site (Chiou et al., 2017). It has subsequently been found to function in the establishment of cell polarity in multiple contexts. For example, Cdc42 recruits and activates the anterior PAR complex to polarise the anterior-posterior axis in the C. elegans zygote and the apical-basal axis during the asymmetric divisions of Drosophila neural stem cells (Gotta et al., 2001; Kay and Hunter, 2001; Atwood et al., 2007; Rodriguez et al., 2017). 
Cdc42 also plays an essential role in the apical-basal polarisation of epithelial cells, where it is required for apical domain formation (Genova et al., 2000; Hutterer et al., 2004; Jaffe et al., 2008; Bray et al., 2011; Fletcher et al., 2012). Cdc42 is active when bound to GTP, which changes its conformation to allow it to bind downstream effector proteins that control the cytoskeleton and membrane trafficking. An important Cdc42 effector in epithelial cells is the Par-6/aPKC complex. Par-6 binds directly to the switch 1 region of Cdc42 GTP through its semi-CRIB domain (Cdc42 and Rac interactive binding) (Lin et al., 2000; Joberty et al., 2000; Qiu et al., 2000; Yamanaka et al., 2001). This induces a change in the conformation of Par-6 that allows it to bind to the C-terminus of another key apical polarity factor, the transmembrane protein, Crumbs, which triggers the activation of aPKC's kinase activity (Dong et al., 2019; Peterson et al., 2004; Whitney et al., 2016). As a result, active aPKC is anchored to the apical membrane, where it phosphorylates and excludes lateral factors, such as Lgl, Par-1 and Bazooka (Betschinger et al., 2003; Hurov et al., 2004; Suzuki et al., 2004; Nagai-Tamai et al., 2002; Morais-de-Sá et al., 2010). In addition to this direct role in apical-basal polarity, Cdc42 also regulates the organisation and activity of the apical cytoskeleton through effectors such as NWASP, which promotes actin polymerisation, and MRCK (Gek in Drosophila), which phosphorylates the myosin regulatory light chain to activate contractility (Padrick and Rosen, 2010; Zihni et al., 2017).

This crucial role of active $\mathrm{Cdc} 42$ in specifying the apical domain raises the question of how Cdc42-GTP itself is localised apically. In principle, this could involve activation by Cdc42GEFs that are themselves apical or lateral inactivation by Cdc42GAPs. The Cdc42GEFs, Tuba, Intersectin 2 and Dbl3 have been implicated in activating Cdc42 in mammalian epithelia (Oda et al., 2014; Otani et al., 2006; Qin et al., 2010; Rodriguez-Fraticelli et al., 2010; Zihni et al., 2014). Only Dbl3 localises apical to tight junctions, however, as Tuba is cytoplasmic and enriched at tricellular junctions and Intersectin 2 localises to centrosomes. Thus, GEF activity may not be exclusively apical, suggesting that it is more important to inhibit Cdc42 laterally. Although nothing is known about the role of GAPs in restricting Cdc42 activity to the apical domain of epithelial cells, this mechanism plays an instructive role in establishing radial polarity in the blastomeres of the early C. elegans embryo. In this system, the Cdc42 GAP, PAC-1, is recruited by the Cadherin adhesion complex to 
sites of cell-cell contact, thereby restricting active Cdc42 and its effector the Par6/aPKC complex to the contact-free surface (Anderson et al., 2008; Klompstra et al., 2015).

Here we analysed the roles of Cdc42GAPs in epithelial polarity using the follicle cells that surround developing Drosophila egg chambers as a model system (Bastock and St Johnston, 2008). By generating mutants in a number of candidate Cdc42 GAPs, we identified the Pac-1 orthologue, RhoGAP19D, as the GAP that restricts active Cdc42 to the apical domain. In the absence of RhoGAP19D, lateral Cdc42 activity leads to an expansion of the apical domain and a high frequency of epithelial invasion into the germline tissue, a phenotype that mimics the early steps of carcinoma formation.

\section{Results}

To confirm that Cdc42 regulates apical domain formation in Drosophila epithelia, we generated homozygous mutant clones of $c d c 42^{2}$, a null allele, in the follicle cell epithelium that surrounds developing egg chambers (Fig 1A). Mutant cells lose their cuboidal shape, leading to gaps and multi-layering in the epithelium, and fail to localise GFP-aPKC apically, indicating that $\mathrm{Cdc} 42$ is required for polarity in the follicle cell layer.

There are 22 Rho GTPase activating proteins in the Drosophila genome (Table S1), but in most cases, it is unclear whether they regulate Rho, Rac or Cdc42. We therefore predicted the tendency for each of the Drosophila GAPs to interact with Cdc42 using InterPReTS (Aloy and Russell, 2002). This uses a known structure of a protein complex (in this case the structure of the human CDC42/ARHGAP1 complex, (Nassar et al., 1998) as a template to predict whether homologous proteins (in this case other Drosophila GAPs and Cdc42) would be able to interact in the same way. The fit of each sequence pair on the structure is assessed via statistical potentials that score the compatibility of each amino acid pair at the (e.g. GAP/Cdc42) interface. The ranks for each Drosophila GAP according to its likelihood of interacting with Drosophila Cdc42 is shown in Table S2 
Interestingly, the Drosophila ARHGAP1 orthologue, RhoGAP68F was only second in the ranking behind another known Cdc42GAP, the PAC-1 orthologue,

RhoGAP19D. Inspection of the InterPreTS results in detail shows that several key, conserved positions mediating interactions in the structure of the human CDC42/ARHGAP1 complex are conserved in RhoGAP19D (and many other) Drosophila GAPs, in addition to three positions that appear to make the interaction stronger (Figure 1). Specifically, an Arg in ARHGAP1 is replaced by a Ile (1237 in RhoGAP19D) making for a better interaction with Ala13 in Cdc42, a Val is replaced by a Ser (1275) making a more favourable interaction with Glu62, and a Thr is replaced by a Lys (1237) possibly making an additional salt bridge with Glu95 and additional interactions with Asn92.

To test whether any of these putative Cdc42 GAPs play a role in epithelial polarity, we generated null mutants in the seven highest ranked GAPs using CRISPR-mediated mutagenesis (Table S3) and examined their phenotypes in the follicle cell epithelium that surrounds developing egg chambers. Mutants in RhoGAP92B are lethal and we therefore used the Flp/FRT system to generate homozygous mutant follicle cell clones, whereas mutants in the other GAPs are homozygous viable or semi-viable, allowing us to analyse the follicle cell phenotype in homozygous mutant females. Null mutants in RhoGAP68F, CdGAPr, RhoGAP92B, RhoGAP82C, conundrum(Neisch et al., 2013) and RhoGAP93B cause no discernible changes in follicle cell shape or polarity, as shown by the localisation of aPKC apically and Lgl laterally (Fig S2). By contrast, 40\% of homozygous mutant RhoGAP19D egg chambers show invasions of regions of the follicular epithelium into the overlying germline cyst (Figure 2A-C). This invasive phenotype is not a consequence of overproliferation of the mutant follicle cells, because mutant egg chambers contain the same number of follicle cells as wild-type egg chambers and the same proportion of homozygous mutant cells and wild-type cells are in mitosis at stages 4-5 (Figure 2 DF). Introducing endogenously tagged E-cadherin into the RhoGap19D mutant background reveals that the invading follicle cells maintain their apical adherens junctions, indicating that they have not undergone an epithelial to mesenchymal transition and are still epithelial in nature (Figure $2 \mathrm{G}$ ). 
We examined the localisation of RhoGAP19D protein by using CRISPR-mediated homologous recombination to insert the $\mathrm{mNeonGreen} \mathrm{fluorescent} \mathrm{tag} \mathrm{at} \mathrm{the} \mathrm{N-}$ terminus of the endogenous RhoGAP19D coding region. Neon::RhoGAP19D localises laterally in the follicle cells, covering the full length of the domain including the apical adherens junctions, where it sometimes appears to be slightly enriched (Figure $2 \mathrm{~h}$ and $\mathrm{i}$ ). A similar lateral localisation is observed in the salivary gland and the testis accessory gland, indicating that RhoGAP19D is a lateral factor in multiple epithelia (Figure S3).

The C. elegans orthologue of RhoGAP19D, PAC-1, is recruited to cell contacts by the E- Cadherin complex through redundant interactions with $\alpha$-catenin and p120-catenin (Klompstra et al., 2015). We observed no change in the lateral recruitment of RhoGAP19D in p120 catenin null mutants, but the junctional signal was almost completely lost when $\alpha$-catenin was depleted by RNAi (Figure 2i-1, Figure SXa). Thus, RhoGAP19D is localised to the lateral membrane by a non-redundant interaction with $\alpha$-catenin, which links it to Cadherin adhesion complexes. The lateral localisation of RhoGAP19D was strongly reduced in clones homozygous for $\operatorname{shotgun}^{\mathrm{IG} 29}$, a null mutant in shotgun (E-cadherin), whereas clones homozygous for a deletion of N-cadherin 1 and N-cadherin 2 had no effect(Tepass et al., 1996; Prakash et al., 2005). The weaker phenotype of $\operatorname{shotgun}^{\mathrm{IG} 29}$ clones compared to $\alpha$-catenin knockdown is presumably because $\mathrm{N}$-cadherin is up-regulated in E-cadherin mutants and either E- or N-Cadherin can recruit $\alpha$-catenin and RhoGAP19D (Grammont, 2007). $\alpha$-catenin and E-cadherin are concentrated in the apical adherens junctions, whereas RhoGAP19D shows only a slight apical enrichment and is much more uniformly distributed along the lateral membrane. Since this suggests that other factors may modulate the recruitment of RhoGAP19, we examined whether any lateral polarity factors affect its distribution, but observed no change when the lateral adhesion proteins, FasII, FasIII or Neuroglian are knocked down by RNAi or in null mutant clones for the lateral polarity factor, Lgl (Fig 2m, Fig S2b-g).

The localisation of RhoGAP19D suggests that it may function to inhibit Cdc42 laterally. We therefore examined where Cdc42 is active by following the localisation 
of an endogenously tagged version of the Cdc42 effector, N-WASP (Kim et al., 2000). In wild-type cells, N-WASP-Neon localises exclusively to the apical domain, consistent with the apical localisation of active Cdc42. By contrast, N-WASP also localizes along the lateral membrane in rhogap19d mutant clones (Fig 3a,b). Thus, RhoGAP19D functions to inhibit Cde42 laterally and is the only GAP that fulfills this role in the follicle cells.

To confirm that RhoGAP19D represses Cdc42 activity, we used UAS- GrabFP-A-int to mislocalise the protein to the apical domain (Harmansa et al., 2017). The GrabFPA-int construct consists of an N-terminal Cherry, a transmembrane domain and an anti-GFP nanobody fused to the localisation signal of Bazooka (Par-3). When this construct is expressed in the follicle cells under the control of Tj-Gal4, the fusion protein localises to the apical membrane and apical junctions without any apparent effect on the appearance of the cells (Fig 3A). Similarly, over-expression of UASGFP-RhoGAP19D alone results in higher levels of RhoGAP19D along the lateral membrane, but has no effect on cell polarity or morphology during stages 1-8 of oogenesis. When GFP-RhoGAP19D and GrabFP-A-int are co-expressed, however, the apical recruitment of GFP- RhoGAP19D by the anti-GFP nanobody disrupts polarity and epithelial organization, as shown by the failure to concentrate aPKC apically and the irregular cell shapes (Fig 3E,F). This phenotype closely resembles that of $c d c 42$ mutants, providing further evidence RhoGAP19D is a specific Cdc42 GAP.

To investigate the cellular basis for the invasive behaviour of rhogap19d mutant follicle cells, we compared the phenotypes of mutant and wildtype cells in the same epithelium by generating homozygous mutant clones. Live imaging reveals that rhogap19d mutant cells are taller than wild-type cells, with dome-shaped apical surfaces that protrude into the germ line (Figure 4A). The distance between the apical and basal surfaces of mutant cells is $20 \%$ greater than wild-type in fixed samples (Figure 4b,e). However, the adherens junctions, marked by E cadherin-GFP and Canoe, do not show a corresponding change in position, and remain level with or slightly below the adherens junctions in the neighbouring wild-type cells (Figure 4b,c, g). The adherens junctions mark the boundary between the apical and lateral domains, suggesting that the apical domain has expanded. This is indeed the case, as GFP- 
aPKC localises all over the domed region of membrane above the adherens junctions, which is $40 \%$ longer than in wild-type. (Figure 4c, f). The apical transmembrane protein Crumbs show a similar extension across the expanded apical domain, but is more enriched in the sub-apical region above the adherens junctions, reflecting its accumulation in regions where it can engage in homophilic interactions with Crumbs in adjacent cells (Thompson et al., 2013) (Figure 4c). By contrast, the lateral domain, marked by Lgl-GFP, is decreased in length (Figure 4d, g). Lateral Cdc42 activity therefore expands the apical domain at the expense of the lateral domain to generate taller cells that protrude into the germ line. A similar apical expansion is also observed in rhogap19d mutant testis accessory glands, suggesting that this is a general phenotype of loss of RhoGAP19D in Drosophila epithelia (Fig S3).

To gain insight into how rhogap19d mutant follicle cells invade the germ line, we imaged living egg chambers at stages 5-7, the stages when invasions are most likely to occur (Movie S1, Figure 5a). Not only are the mutant cells taller than wild-type with domed apical surfaces, but they are also more motile. Temporal projections show that the mutant cells expand and contract along their apical-basal axes, whereas wildtype cells are static (Figure 5b). The apical expansion of the mutant cells and the up and down movements are likely to increase strain in the epithelium and raise the probability of regions of the follicle cell layer buckling towards the germ line (Figure 5a). More rarely, we observe clusters of cells that have detached from the basement membrane and are beginning to invade (Figure $5 \mathrm{c}$ ).

The higher motility suggests that myosin activity is increased in mutant cells, and we therefore examined the distribution of non-muscle myosin II (NMYII) using a protein trap insertion in the heavy chain (Zipper). This revealed that the mutant cells have more numerous and larger NMYII foci along their lateral membranes and reduced levels of apical NMYII (Figure 5d). This increase in lateral NMYII is likely to account for the apical-basal contractions in mutant cells. In MDCK cells, Cdc42 recruits and activates NMYII apically through its effector, Myotonic dystrophy kinase-related Cdc42-binding kinase (MRCK), which phosphorylates the myosin regulatory light chain to stimulate contractility (Zihni et al., 2017; Zhao and Manser, 2015). This suggests that the Drosophila orthologue of MRCK, Genghis kan (Gek) 
might play a similar role in coupling Cdc42 to the activation of NMYII in the follicle cell epithelium. Antibody staining revealed that Gek is predominantly localised to the apical surface of the follicle cells, consistent with its role in MDCK cells (Figure 5e). In rhogap 19d mutants, however, Gek extends along the lateral membrane (Figure 5f). Thus, the ectopic Cdc42 activity in rhogap19d mutants recruits Gek to the lateral membrane, where it can localise and activate NMYII.

Our results suggest that the invasive phenotype of rhogap 19d mutants depends on a partial disruption of polarity, in which the apical domain expands at the expense of the lateral domain. Since the relative sizes of the apical and lateral domains are determined by mutual antagonism between apical and lateral polarity factors, reducing the dosage of lateral factors should enhance this phenotype, whereas reducing apical factors should suppress it. We therefore tested whether polarity mutants act as dominant modifiers of the rhogap19d phenotype (Figure 6). Removing one copy of the lateral polarity proteins, $\operatorname{lgl}$ and $s c r i b$, doubles the frequency of germline invasion, as does removing both copies of Fasciclin II or RNAi knock down Neuroglian, both of which are the lateral adhesion factors (Bilder and Perrimon, 2000; Wei et al., 2004; Szafranski and Goode, 2006). By contrast, loss of one copy of $a P K C$ or $c r b$ strongly suppresses invasion. Reducing the dosage of gek also decreases the frequency of invasion, consistent with a role for Gek in activating of NMYII laterally to stimulate the movement of the follicle cells into the germ line. Thus, these genetic interactions support the view that the invasive behaviour of rhogap $19 d$ mutant cells is driven by the expansion of the apical domain and Gek-dependent lateral contractility, both of which will increase the stress on the epithelium and promote buckling, without completely disrupting polarity.

Two of the mutants showed unexpected genetic interactions with rhogap19d. Firstly, reducing the dosage of the lateral polarity factor, Par-1, suppressed the invasive phenotype of the rhogap19d mutant, whereas the other lateral factors strongly enhance it. Par-1 localises to the lateral membrane and functions to limit the basal extent of the adherens junctions by phosphorylating and antagonising Bazooka (Par-

3) (Benton and St Johnston, 2003; Wang et al., 2012). The ability of the par-1 mutant to suppress rhogap19d indicates that Par-1 does not function in the same pathway as Scrib, Lgl, FasII and Nrg and suggests instead that it either negatively regulates these 
lateral factors or positively regulates apical ones. Secondly, Pak1 has been reported to function redundantly with aPKC to specify the apical domain downstream of active Cdc42 (Aguilar-Aragon et al., 2018). Although one would therefore expect the pak1 mutant to suppress the invasive phenotype like mutants in the other in apical factors, it acts a strong enhancer of invasion. This is consistent with the role of Pak1 as a component of the lateral Scribbled complex and argues against the proposal that it functions as an apical Cdc42 effector kinase (Bahri et al., 2010).

\section{Discussion}

Here we report that RhoGAP19D restricts Cdc42 activity to the apical side of the follicle cells and probably many other Drosophila epithelial tissues. In the absence of RhoGAP19D, both N-Wasp and Gek are recruited to the lateral membrane, indicating that Cdc42 is ectopically activated there. This implies that RhoGAP19D is the sole Cdc42GAP that represses Cdc42 laterally. This also suggests that the GEFs that activate $\mathrm{Cdc} 42$ are not restricted to the apical domain and can turn it on laterally once this repression is removed. This is consistent with the identification of multiple vertebrate GEFs with different localisations that contribute to apical $\mathrm{Cdc} 42$ activation (Oda et al., 2014; Otani et al., 2006; Qin et al., 2010; Rodriguez-Fraticelli et al., 2010; Zihni et al., 2014). Our results therefore identify RhoGAP19D as a new lateral polarity factor. This leads to a revised network of polarity protein interactions, in which RhoGAP19D functions as the third lateral factor that antagonizes the activity of apical factors, alongside Lgl, which inhibits aPKC, and Par-1 which excludes Bazooka/Par-3 (Wirtz-Peitz et al., 2008; Benton and St Johnston, 2003)(Fig 7a)

The function of RhoGAP19D is very similar to that of its orthologue PAC-1, which inhibits Cdc42 at sites of cell contact in early C. elegans blastomeres to generate distinct apical and basolateral domains (Anderson et al., 2008). Both RhoGAP19D and PAC-1 are recruited to the lateral domain by E-cadherin complexes, although the exact mechanism is slightly different. RhoGAP19D recruitment is strictly dependent on $\alpha$-catenin, which links it through $\beta$-catenin to the E-cadherin cytoplasmic tail, whereas $\alpha$-catenin (HMP-1) and p120-catenin (JAC-1) play partially redundant roles in recruiting PAC-1 to E-cadherin (HMR-1) in the worm (Klompstra et al., 2015). Nevertheless, in both cases, the recruitment of the Cdc42GAP translates the spatial 
cue provided by the localization of cadherin to sites of cell-cell contact into a polarity signal that distinguishes the lateral from the apical domain. Classic work on the establishment of polarity MDCK cells grown in suspension has revealed that the recruitment of cadherin (uvomorulin) to sites of cell-cell contact is the primary cue that drives the segregation of apical proteins from basolateral proteins (Wang et al., 1990). Furthermore, the expression of E-cadherin in unpolarized mesenchymal cells is sufficient to induce this segregation, although the mechanisms behind this process are only partially understood (Wang et al., 1990; McNeill et al., 1990; Watabe et al., 1994; Nejsum and Nelson, 2007). Our observation that RhoGAP19D directly links cadherin adhesion to the polarity system in epithelial cells extends the results of Klompstra et al. (2015) in early blastomeres, strongly suggesting that PAC1/RhoGAP19D plays an important role in the first steps in epithelial polarization.

Although PAC-1 and RhoGAP19D perform equivalent functions in early blastomeres and epithelial cells, there is one important difference between their mutant phenotypes. In pac-1 mutants, Par- 6 and aPKC are mislocalised to the contacting surfaces of C. elegans blastomeres where Cdc42 is ectopically active(Anderson et al., 2008). By contrast, Par-6 and aPKC are not mislocalised laterally in rhogap19d mutant Drosophila epithelial cells, even though lateral Cdc42-GTP does recruit two other Cdc42 effectors, N-Wasp and GEK. Thus, lateral Cdc42 activity is sufficient to recruit Par-6/aPKC to the lateral domain in early blastomeres, but not in epithelial cells. Instead, we observe that lateral Cdc42 activity in rhogap 19d mutant follicle cells acts at a distance to expand the size of the apical domain. A likely explanation for this difference is the presence of Crumbs in epithelial cells. The interaction between Cdc42-GTP and Par-6 alters the conformation of Par-6 so that it can bind to Crumbs, which anchors the Par-6/aPKC complex to the apical membrane and activates aPKC's kinase activity (Peterson et al., 2004; Whitney et al., 2016; Dong et al., 2020). Although Par-6 presumably binds to Cdc42 laterally in rhogap19D mutants and undergoes the conformational change, it cannot be anchored laterally in the absence of Crumbs. This activated Par-6/aPKC complex can then diffuse until it is captured by Crumbs in the apical domain, thereby increasing apical aPKC activity, providing an explanation for why the apical domain expands in rhogap $19 d$ mutant cells (Figure 7b). C. elegans has three Crumbs orthologues, but removal of all three 
simultaneously has no effect on viability or polarity (Waaijers et al., 2015). Thus, in contrast to epithelial cells, C. elegans Crumbs proteins are not required for Par6/aPKC localisation and activation, suggesting that some other mechanism, such as $\mathrm{Cdc} 42$ binding is sufficient to activate aPKC.

If the failure of active Cdc42 to recruit aPKC laterally in rhogap19d mutant cells is due to the absence of Crumbs in this region, there must be a mechanism to exclude Crumbs from the lateral domain. One proposed mechanism depends on Yurt (Moe and EPB41L5 in vertebrates), which is restricted to the lateral domain by aPKC and binds to Crumbs to antagonize its activity (Laprise et al., 2006). However, we did not observe any lateral recruitment of aPKC in rhogap19d; yurt double mutant cells. Thus, there must be some parallel mechanism that excludes Crumbs, Par-6 and/or aPKC from the lateral domain.

Although loss of RhoGAP19D only leads to a partial disruption of polarity, it causes the follicular epithelium to invade the adjacent germline tissue with $40 \%$ penetrance. This invasive behaviour is not driven by an epithelial to mesenchymal transition, as the cells retain their apical adherens junctions and epithelial organisation. Instead, the deformation of the epithelium seems to be driven by the combination of an increase in lateral contractility and an expansion of the apical domain, as reducing the dosage of Gek, which activates myosin II to drive the contractility, significantly reduces the frequency of this phenotype, as does halving the dosage of any of the apical polarity factors. The invasion seems most likely to be driven by the buckling of the epithelium. Recent work has shown that epithelial monolayers under compressive stress and constrained by a rigid external scaffold have a tendency to buckle inwards (Trushko et al., 2020). The follicle cell layer is surrounded by an extracellular matrix that constrains the shape of the egg chamber and which should therefore resist expansion (Haigo and Bilder, 2011). Furthermore, the expansion of the apical domain should generate compressive stress because the domain is too long for the cells to adopt the lowest energy conformation while remaining in the epithelium. The lateral contractility may also contribute to compressive stress because reducing cell height while maintaining a constant volume will exert a pushing force on the neighbouring cells. In support of this view, lateral contractility has been shown to drive the folding of the imaginal wing disc between the prospective hinge region and the pouch (Sui et 
al., 2018). This phenotype therefore provides an example of how a partial disruption of polarity can induce cell shape changes that lead to major alterations in tissue morphogenesis (St Johnston and Sanson, 2011).

The rhogap19d phenotype resembles the defects earliest observed in the development of ductal carcinoma in situ (DCIS) (Halaoui et al., 2017). In flat epithelial atypia (FEA), the ductal cells are still organized into an epithelial layer, but they display apical protrusions that are strongly labeled by the apical polarity factor, Par- 6 . This suggests that the apical domain has expanded and bulges out of the cell, just as we observe in the rhogap19d mutant follicle cells. In the next stage, atypical ductal hyperplasia $(\mathrm{ADH})$, the ductal cells start to invade the lumen of the duct, while retaining aspects of normal apical-basal polarity, again resembling the invasive phenotype reported here (Fig S4). Thus, these abnormalities, which can sometimes progress to DCIS and breast cancer, mirror the effects of lateral Cdc42 activation. The RhoGAP19D humans orthologues, ARHGAP21 and 23, has been shown to bind directly to $\alpha$-catenin and localize to cell-cell junctions (Sousa et al., 2005) (Van Itallie et al., 2014). Furthermore, low expression of ARHGAP21 or 23 correlates with reduced survival rates in several cancers of epithelial origin (Fig S4) (Györffy et al., 2010). It would therefore be interesting to determine whether these orthologues perform the same functions in epithelial polarity as RhoGAP19D and if their loss contributes to tumour development.

\section{Materials \& Methods}

\section{Predicting Cdc42/GAP interactions:}

We identified and aligned putative Drosophila GAPs by searching the Pfam (Finn et al., 2016) hidden Markov model (HMM) profile PF00620.26 (RhoGAP) against the 
UniProt Drosophila melanogaster proteome using HMMsearch (Eddy, 2009). We aligned significantly scoring sequences together with human ARHGAP1 (from the 3D structure RCSB entry 1 grn) using HMMalign (Eddy, 2009).

We scored the potential interaction of each Drosophila GAP/Cdc42 pair using the structure of human ARHGAP1/CDC42 (RCSB 1grn:B/A) via InterPReTS (Aloy \& Russell, 2002), which assesses the effect of evolutionary changes at the interface structure using empirical pair potentials (Betts et al., 2015).

We modelled the Cdc42-RhoGAP19D 3D complex using SwissModel (Waterhouse et al., 2018) and rendered the interaction interface using the PyMOL Molecular Graphics System, Version 2.3.0, Schrödinger, LLC.

\section{Drosophila mutant stocks and transgenic lines:}

We used the following mutant alleles and transgenic constructs: $c d c 42^{2}$ ((Fehon et al.,

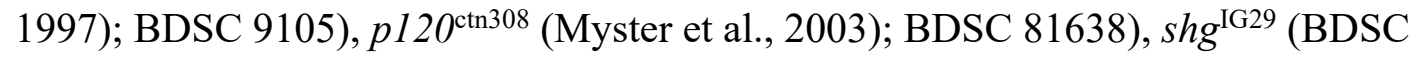
58471), $\lg l^{4}$ (Gateff, 1978); BDSC 36289), $s c r i b^{2}$ (Bilder and Perrimon, 2000), fasII ${ }^{\mathrm{G} 0336}$ (Mao and Freeman, 2009); Kyoto 111871), par-1 ${ }^{6323}$ (Shulman et al., 2000), $a P K C^{\mathrm{HC}}$ (Chen et al., 2018), $c r b^{8 \mathrm{~F} 105}$ (Tepass et al., 1990), gek ${ }^{\mathrm{omb} 1080}$ (Gontang et al., 2011), Pakl $^{22}$ (Newsome et al., 2000); a gift from Dickson lab), $\mathrm{CadN}^{\Delta_{14}}$ (Prakash et al., 2005), $\alpha$-catenin RNAi (BDSC 33430), nrg RNAi (BDSC 38215), fasII RNAi (BDSC 34084), fasIII RNAi (BDSC 77396), scrib RNAi (BDSC 35748), cora RNAi (VDRC 9788), rhogap19d RNAi (P \{TRiP.HMS00352\}attP2; BDSC 32361), E-cadEGFP (BDCS 60584; (Huang et al., 2009)), Lgl-EGFP (Tian and Deng, 2008), mNeonGreen-NWasp (a gift from Jenny Gallop, The Gurdon Institute), mCherryGrabFP-Baz (Harmansa et al., 2017), aPKC-EGFP (Chen et al., 2018), Zipper-EGFP (Lowe et al., 2014), UASp-GFP RhoGAP19D (BDSC 66167), , y* w*; P \{GawB\}NP1624 (Traffic Jam-Gal4; (Brand and Perrimon, 1993)), nos-GAL4 (a gift from Ruth Lehmann). The following stocks were used to generate mitotic clones: ubiRFP-nls, hsflp, FRT20A4 (PBac\{WH\} f01417, Exelixis), FRT40A ubiRFP-nls (BDSC 34500), FRT82B, ubiRFP-nls (BDSC 30555) and FRT82B ubiGFP (BDSC 5188), y w hs-FLP;; Act5C >CD2>Gal4, UAS:mRFPnls ((Pignoni and Zipursky, 1997); BDSC 30558).

\section{Generation of endogenously tagged RhoGAP19d}


The mNeonGreen tag (Shaner et al., 2013) was fused to the N-terminus of RhoGAP19d by CRISPR-mediated homologous recombination. In vitro synthesised gRNA to a CRISPR target (target sequence GGTGGCGACTCCGGCAGCGGCGG, CRISPR; loc. $25985 \mathrm{bp}$ from the 5' end) and a plasmid donor containing the ORF of $\mathrm{mNeonGreen} \mathrm{as} \mathrm{well} \mathrm{as} \mathrm{appropriate} \mathrm{homology} \mathrm{arms} \mathrm{(1.5} \mathrm{kb} \mathrm{upstream} \mathrm{and}$ downstream) were co-injected into nos-Cas9-expressing embryos (BDSC 54591). Single F0 flies were mated to $\mathrm{y} w$ flies and allowed to produce larvae before the parents were analysed by PCR. Progeny from F0 flies in which a recombination event occurred (as verified by PCR) were crossed and sequenced to confirm correct integration. Several independent mNeonGreen-RhoGAP19d lines were isolated. Recombinants carry the $\mathrm{mNeonGreen} \mathrm{coding} \mathrm{sequence} \mathrm{inserted} \mathrm{immediately}$ downstream of the endogenous start codon with a short linker (Gly-Ser-Gly-Ser) between the coding sequence of mNeonGreen and the coding sequence of RhoGAP19d. Homozygous flies are viable and healthy.

To discriminate between the signal of mNeonGreen-RhoGAP19D in the germline and in the somatic follicle cells, UAS-RhoGAP19D RNAi was expressed in the germline under the control of nos-Gal4. The knock-down was efficient, which allowed visualisation Neon-RhGAP19D expression in the follicle cells only.

\section{Generation of rhogap19d mutant flies}

We used the error prone repair of CRISPR/Cas9-induced dsDNA breaks by the nonhomologous end joining repair pathway to generate null alleles of rhogap19d (Bassett et al., 2013). The following target sequence was used GGGTCGGGATCCCTTTCGGGGGG, CRISPR (38613bp from 5' end). In vitro synthetized gRNA to the target sequence and Cas9 mRNA were injected into FRT20A4 embryos. Single F0 flies were mated to y w flies. DNA from F0 flies was extracted and analysed by High Resolution Melting (HRM). Short fragments of about $150 \mathrm{bp}$ covering the region containing the target sequence were amplified by PCR. Slow melting curves were generated for the PCR products and changes in sequence were measured by changes in fluorescence as the strands separate. This technique allows the detection of single base changes. Progeny of promising F0 candidates were balanced, and sequenced. Multiple mutant alleles of rhogap19d on the FRT20A4 chromosome were isolated and analysed. The mutants that contain insertions or 
deletions generating premature STOP codons were kept for clonal analysis. These mutations generate proteins of $\sim 436$ amino acids that lack the RhoGAP domain, but contain the PDZ domain. rhogap19d mutant flies are semi-lethal. The follicle cell phenotypes of the rhogap $19 d$ alleles used in this study are rescued by the expression of UAS-RhoGAP19D-GFP under the control of TJ-Gal4, confirming that they are caused by loss of RhoGAP19D function.

\section{Reagents:}

The following antibodies were used: Primary antibodies: anti-Armadillo (N2 7A1, Developmental Studies Hybridoma Bank (DSHB) 1:100 dilution), anti-aPKC (C-20, sc-216-G, Santa Cruz, goat polyclonal IgG, 1:500), anti-Cno ((Takahashi et al., 1998); a gift from M. Peifer, University of North Carolina, USA, 1:1,000 dilution) anti-Gek ((Gontang et al., 2011); a gift from the Clandinin lab,1:25 dilution), anti-PH3 (Cell Signalling \#9701S, 1:500 dilution).

Secondary antibodies: Alexa Fluor secondary antibodies (Invitrogen) were used at a dilution of 1:1,000. Alexa Fluor 488 goat anti-mouse (\#A11029), Alexa Fluor 488 goat anti-rabbit (\#A11034), Alexa Fluor 647 goat anti-mouse (\#A21236), Alexa Fluor 647 goat anti-rabbit (\#A21245).

F-Actin was stained with phalloidin conjugated to Rhodamine (Invitrogen, Cat. \#R415, 1:500 dilution). The cell membranes were labelled with CellMask Orange Plasma Membrane Stain or CellMask Deep Red Plasma Membrane Stain (Thermo Fisher Scientific).

\section{Immunostaining:}

Ovaries from fattened adult females, salivary glands from L3 instar larvae and accessory glands from virgin males were dissected in Phosphate-buffered Saline (PBS) and fixed with rotation for $20 \mathrm{~min}$ in $4 \%$ paraformaldehyde and $0.2 \%$ Tween 20 in PBS. After a few washes with PBS-0.2\%Tween, tissues were then incubated in $10 \%$ bovine serum albumin (BSA) in PBS to block for at least 1 hour at room temperature. Incubations with primary antibodies were performed at $4{ }^{\circ} \mathrm{C}$ overnight in PBS, $0.2 \%$ Tween 20 and 1\% BSA. This step was followed by four washes with PBS$0.2 \%$ Tween and samples were then incubated for 3-4 h with secondary antibody at room temperature. Specimens were then washed several times in washing buffer and mounted in Vectashield containing DAPI (Vector Laboratories). 
For stainings with the anti-Gek antibody, ovaries were heat fixed as described by (Chen et al., 2018).

\section{Imaging:}

Fixed samples and live imaging was performed using an Olympus IX81 (40×/1.3UPlan FLN Oil or 60×/1.35 UPlanSApo Oil) or a Leica SP8 white Laser (63×/1.4 HC PL Apo CS Oil) inverted confocal microscope. For live observations, ovaries were dissected and imaged in 10S Voltalef oil (VWR Chemicals). Images were processed with Fiji (Schindelin et al., 2012) or Leica analysis software.

\section{Drosophila genetics}

Standard procedures were used for Drosophila maintenance and experiments. Flies were grown on standard fly food supplemented with live yeast at $25^{\circ} \mathrm{C}$. Follicle cell clones were induced by incubating larvae or pupae at $37^{\circ} \mathrm{C}$ for 2 hours every 12 hours over a period of at least 3 days. Adult females were dissected at least 2 days after the last heat shock. In some experiments adult flies were heat-shocked for at least 3 days and dissected one day after the last heat shock.

We used the Flipout technique with Actin $5 \mathrm{c}>\mathrm{Cd} 2>\mathrm{Gal} 4$ to generate marked clones of cell expressing RNAi constructs (Fig. S3). Flp recombination was induced by incubating larvae or pupae at $37^{\circ} \mathrm{C}$ for 2 hours every 12 hours over a period of at least 3 days.

\section{Genetic interactions:}

To test for genetic interactions between rhogap 19d and adhesion molecules or polarity factors, we analysed the frequency of follicle cell invasions at stages 7 and 8 in large anterior rhogap 19d clones that covered at least $25 \%$ of the follicular epithelium in each genetic background.

An unpaired, two-tailed students t-test with Welch's correction was used to determine whether any differences between the penetrance of the invasion phenotype in rhogap19d alone and in combination with each mutant or RNAi knockdown were significant. 
Confocal z-stacks of whole egg chambers were collected on a Leica SP8 white laser microscope. Each egg chamber was divided in three regions. Nuclei were counted twice per region.

\section{Reproducibility of experiments:}

All experiments were repeated multiple times as listed below. For each figure, the first number indicates the number of times that the experiment was repeated and the second indicates the number of egg chambers or clones analysed. The number of independent experiments performed were: Figure 1a $(3 ; 28)$, Figure $2 \mathrm{c}(5 ; 89)$, Figure $2 \mathrm{e}(3 ; 34)$, Figure $2 \mathrm{f}(2 ; 20)$, Figure $2 \mathrm{~h}(7 ; 56)$, Figure $2 \mathrm{i}(4 ; 34)$, Figure $2 \mathrm{j} \& \mathrm{k}(3 ; 6)$, Figure $21(2 ; 9)$, Figure $2 \mathrm{~m}(3 ; 12)$, Figure $2 \mathrm{n}(2 ; 7)$. Figure $3 \mathrm{a}(4 ; 24)$, Figure $3 \mathrm{~b}(3$; 46). Figure $4 \mathrm{a}(7 ; 67)$, Figure $4 \mathrm{~b}(3 ; 78)$, Figure $4 \mathrm{c}(5 ; 98)$, Figure $4 \mathrm{~d}(2 ; 16)$, Figure 4e $(3 ; 56)$. Figure 5a $(7,56)$, Figure $5 \mathrm{c}(5 ; 47)$, Figure 5d $(3 ; 98)$, Figure 5e $(3 ; 18)$. Figure 6: rhogap19d (8; 301), scrib/+ (3; 194), $\operatorname{lgl} /+(3 ; 94)$, fas $2(3 ; 185), \operatorname{nrg}(3$; 114), parl/+ (3; 155), aPKC/+ (4; 98), crb (2; 129), gek (2; 78), Pakl (3; 228).

\section{Supplemental Information}

Table S1: List of CRISPR-mediated mutations in candidate Cdc42 GAPS

Figure S1: RhoGAP92B, RhoGAP68F, CdGAPr, RacGAP84C, Conu and RhoGAP93B are not required for follicle cell polarity.

Figure S2: RhoGAP19D localises laterally in multiple epithelia.

Figure S3: Mutants that do not affect the localisation of RhoGAP19D

Figure S4: The rhogap19d phenotype resembles the early steps in breast cancer.

Movie: S1: A time-lapse movie of a stage 7 egg chamber containing a large rhogap 19d mutant clone (marked by the loss of RFP; magenta) and expressing GFPaPKC (green). Frames were captured every 15s. Elapsed time, 11 mins.

\section{Acknowledgements}

We thank Jenny Gallop, Franck Pichaud, Barry Dickson, Marc Peifer, Thomas Clandinin, Ruth Lehmann, Andrea Brand and their labs for fly stocks and for antibodies; the Developmental Studies Hybridoma Bank (DSHB), the Kyoto Stock Center (DGRC), the Vienna Drosophila Resource Center (VDRC), Exelixis, and the Bloomington Drosophila Stock Center (BDSC), Santa Cruz Biotechnology for antibodies and fly stocks; Jia Chen for help with heat fixation, Richard Butler for help 
with image analysis, Nick Lowe for help with HRM analysis and members of the D.St J. lab for technical assistance, helpful comments and criticism. RBR acknowledges support from the German Network of Bioinformatics Infrastructure (de.NBI) funded by the Bundesministerium für Bildung und Forschung (BMBF).

\section{Conflict of interest:}

The authors declare no conflicts of interest.

\section{Author contributions:}

The project was conceived by W.F., R.B., R.B.R. and D.St J. The structural modelling of Drosophila RhoGAP proteins was performed by F.R and R.B.R. The Drosophila experiments were performed by W.F, R.B. and E.L. and the data were analysed by W.F, R.B. and D. St J. The mNeonGreenWASP line was generated by Y.I. and J.G. The project funding, administration and supervision were provided by D. St J. and R.B.R. W.F and D. St J. prepared the figures and wrote the manuscript, which was edited and reviewed by all authors. 


\section{References:}

Aguilar-Aragon, M., A. Elbediwy, V. Foglizzo, G.C. Fletcher, V.S.W. Li, and B.J. Thompson. 2018. Pak1 kinase maintains apical membrane identity in epithelia. Cell Rep. 22:1639-1646. doi:10.1016/j.celrep.2018.01.060.

Aloy, P., and R.B. Russell. 2002. Interrogating protein interaction networks through structural biology. Proc Natl Acad Sci U S A. 99:5896-5901. doi:10.1073/pnas.092147999.

Anderson, D.C., J.S. Gill, R.M. Cinalli, and J. Nance. 2008. Polarization of the $C$. elegans embryo by RhoGAP-mediated exclusion of PAR-6 from cell contacts. Science. 320:1771-. doi:10.1126/science.1156063.

Atwood, S.X., C. Chabu, R.R. Penkert, C.Q. Doe, and K.E. Prehoda. 2007. Cdc42 acts downstream of Bazooka to regulate neuroblast polarity through Par-6 aPKC. J Cell Sci. 120:3200-3206. doi:10.1242/jcs.014902.

Bahri, S., S. Wang, R. Conder, J. Choy, S. Vlachos, K. Dong, C. Merino, S. Sigrist, C. Molnar, X. Yang, E. Manser, and N. Harden. 2010. The leading edge during dorsal closure as a model for epithelial plasticity: Pak is required for recruitment of the Scribble complex and septate junction formation. Development. 137:20232032. doi:10.1242/dev.045088.

Bassett, A.R., C. Tibbit, C.P. Ponting, and J.-L. Liu. 2013. Highly efficient targeted mutagenesis of Drosophila with the CRISPR/Cas9 system. Cell Rep. 4:220-228. doi:10.1016/j.celrep.2013.06.020.

Bastock, R., and D. St Johnston. 2008. Drosophila oogenesis. Curr Biol. 18:R1082-7. doi:10.1016/j.cub.2008.09.011.

Benton, R., and D. St Johnston. 2003. Drosophila PAR-1 and 14-3-3 inhibit Bazooka/PAR-3 to establish complementary cortical domains in polarized cells. Cell. 115:691-704.

Betschinger, J., K. Mechtler, and J. Knoblich. 2003. The Par complex directs asymmetric cell division by phosphorylating the cytoskeletal protein Lgl. Nature. 422:326-330.

Betts, M.J., Q. Lu, Y. Jiang, A. Drusko, O. Wichmann, M. Utz, I.A. ValtierraGutiérrez, M. Schlesner, N. Jaeger, D.T. Jones, S. Pfister, P. Lichter, R. Eils, R. Siebert, P. Bork, G. Apic, A.-C. Gavin, and R.B. Russell. 2015. Mechismo: predicting the mechanistic impact of mutations and modifications on molecular interactions. Nucleic Acids Res. 43:e10-e10. doi:10.1093/nar/gku1094.

Bilder, D., and N. Perrimon. 2000. Localization of apical epithelial determinants by the basolateral PDZ protein Scribble. Nature. 403:676-680. doi:10.1038/35001108.

Brand, A.H., and N. Perrimon. 1993. Targeted gene expression as a means of altering cell fates and generating dominant phenotypes. Development. 118:401-415. 
Bray, K., C. Brakebusch, and T. Vargo-Gogola. 2011. The Rho GTPase Cdc42 is required for primary mammary epithelial cell morphogenesis in vitro. Small GTPases. 2:247-258. doi:10.4161/sgtp.2.5.18163.

Chen, J., A.-C. Sayadian, N. Lowe, H.E. Lovegrove, and D. St Johnston. 2018. An alternative mode of epithelial polarity in the Drosophila midgut. PLoS Biol. 16:e3000041. doi:10.1371/journal.pbio.3000041.

Chiou, J.-G., M.K. Balasubramanian, and D.J. Lew. 2017. Cell polarity in yeast. Annu Rev Cell Dev Biol. 33:annurev-cellbio-100616-060856. doi:10.1146/annurevcellbio-100616-060856.

Dong, W., J. Lu, X. Zhang, Y. Wu, K. Lettieri, G.R. Hammond, and Y. Hong. 2020. A polybasic domain in aPKC mediates Par6-dependent control of membrane targeting and kinase activity. J Cell Biol. 219:1. doi:10.1083/jcb.201903031.

Eddy, S.R. 2009. A new generation of homology search tools based on probabilistic inference. Genome Inform. 23:205-211.

Fehon, R.G., T. Oren, D.R. LaJeunesse, T.E. Melby, and B.M. McCartney. 1997. Isolation of mutations in the Drosophila homologues of the human Neurofibromatosis 2 and yeast CDC42 genes using a simple and efficient reversegenetic method. Genetics. 146:245-252.

Finn, R.D., P. Coggill, R.Y. Eberhardt, S.R. Eddy, J. Mistry, A.L. Mitchell, S.C. Potter, M. Punta, M. Qureshi, A. Sangrador-Vegas, G.A. Salazar, J. Tate, and A. Bateman. 2016. The Pfam protein families database: towards a more sustainable future. Nucleic Acids Res. 44:D279-85. doi:10.1093/nar/gkv1344.

Fletcher, G.C., E.P. Lucas, R. Brain, A. Tournier, and B.J. Thompson. 2012. Positive feedback and mutual antagonism combine to polarize Crumbs in the Drosophila follicle cell epithelium. Curr Biol. doi:10.1016/j.cub.2012.04.020.

Gateff, E. 1978. Malignant neoplasms of genetic origin in Drosophila melanogaster. Science. 200:1448-1459. doi:10.1126/science.96525.

Genova, J.L., S. Jong, J.T. Camp, and R.G. Fehon. 2000. Functional analysis of Cdc42 in actin filament assembly, epithelial morphogenesis, and cell signaling during Drosophila development. Dev Biol. 221:181-194. doi:10.1006/dbio.2000.9671.

Gontang, A.C., J.J. Hwa, J.D. Mast, T. Schwabe, and T.R. Clandinin. 2011. The cytoskeletal regulator Genghis khan is required for columnar target specificity in the Drosophila visual system. Development. 138:4899-4909. doi:10.1242/dev.069930.

Gotta, M., M.C. Abraham, and J. Ahringer. 2001. CDC-42 controls early cell polarity and spindle orientation in C. elegans. Curr Biol. 11:482-488.

Grammont, M. 2007. Adherens junction remodeling by the Notch pathway in Drosophila melanogaster oogenesis. J Cell Biol. 177:139-150. doi:10.1083/jcb.200609079. 
Györffy, B., A. Lanczky, A.C. Eklund, C. Denkert, J. Budczies, Q. Li, and Z. Szallasi. 2010. An online survival analysis tool to rapidly assess the effect of 22,277 genes on breast cancer prognosis using microarray data of 1,809 patients. Breast Cancer Res Treat. 123:725-731. doi:10.1007/s10549-009-0674-9.

Haigo, S.L., and D. Bilder. 2011. Global tissue revolutions in a morphogenetic movement controlling elongation. Science. 331:1071-1074. doi:10.1126/science.1199424.

Halaoui, R., C. Rejon, S.J. Chatterjee, J. Szymborski, S. Meterissian, W.J. Muller, A. Omeroglu, and L. McCaffrey. 2017. Progressive polarity loss and luminal collapse disrupt tissue organization in carcinoma. Genes Dev. 31:1573-1587. doi:10.1101/gad.300566.117.

Harmansa, S., I. Alborelli, D. Bieli, E. Caussinus, and M. Affolter. 2017. A nanobody-based toolset to investigate the role of protein localization and dispersal in Drosophila. Elife. 6:99. doi:10.7554/eLife.22549.

Huang, J., W. Zhou, W. Dong, A.M. Watson, and Y. Hong. 2009. Directed, efficient, and versatile modifications of the Drosophila genome by genomic engineering. Proc Natl Acad Sci USA. 106:8284-8289. doi:10.1073/pnas.0900641106.

Hurov, J.B., J.L. Watkins, and H. Piwnica-Worms. 2004. Atypical PKC phosphorylates PAR-1 kinases to regulate localization and activity. Curr Biol. 14:736-741. doi:10.1016/j.cub.2004.04.007.

Hutterer, A., J. Betschinger, M. Petronczki, and J.A. Knoblich. 2004. Sequential roles of Cdc42, Par-6, aPKC, and Lgl in the establishment of epithelial polarity during Drosophila embryogenesis. Dev Cell. 6:845-854. doi:10.1016/j.devcel.2004.05.003.

Jaffe, A.B., N. Kaji, J. Durgan, and A. Hall. 2008. Cdc42 controls spindle orientation to position the apical surface during epithelial morphogenesis. 183:625-633. doi:10.1083/jcb.200807121.

Joberty, G., C. Petersen, L. Gao, and I. Macara. 2000. The cell-polarity protein Par6 links Par3 and atypical protein kinase C to Cde42. Nat Cell Biol. 2:531-9.

Kay, A.J., and C.P. Hunter. 2001. CDC-42 regulates PAR protein localization and function to control cellular and embryonic polarity in C. elegans. Curr Biol. 11:474-481. doi:10.1016/s0960-9822(01)00141-5.

Kim, A.S., L.T. Kakalis, N. Abdul-Manan, G.A. Liu, and M.K. Rosen. 2000. Autoinhibition and activation mechanisms of the Wiskott-Aldrich syndrome protein. Nature. 404:151-158. doi:10.1038/35004513.

Klompstra, D., D.C. Anderson, J.Y. Yeh, Y. Zilberman, and J. Nance. 2015. An instructive role for $C$. elegans E-cadherin in translating cell contact cues into cortical polarity. Nat Cell Biol. doi:10.1038/ncb3168.

Laprise, P., S. Beronja, N.F. Silva-Gagliardi, M. Pellikka, A.M. Jensen, C.J. McGlade, and U. Tepass. 2006. The FERM protein Yurt is a negative regulatory 
component of the Crumbs complex that controls epithelial polarity and apical membrane size. Dev Cell. 11:363-374. doi:10.1016/j.devcel.2006.06.001.

Lin, D., A.S. Edwards, J.P. Fawcett, G. Mbamalu, J.D. Scott, and T. Pawson. 2000. A mammalian PAR-3-PAR-6 complex implicated in Cdc42/Rac1 and aPKC signalling and cell polarity. Nature Cell Biol. 2:540-547. doi:10.1038/35019582.

Lowe, N., J.S. Rees, J. Roote, E. Ryder, I.M. Armean, G. Johnson, E. Drummond, H. Spriggs, J. Drummond, J.P. Magbanua, H. Naylor, B. Sanson, R. Bastock, S. Huelsmann, V. Trovisco, M. Landgraf, S. Knowles-Barley, J.D. Armstrong, H. White-Cooper, C. Hansen, R.G. Phillips, UK Drosophila Protein Trap Screening Consortium, K.S. Lilley, S. Russell, and D. St Johnston. 2014. Analysis of the expression patterns, subcellular localisations and interaction partners of Drosophila proteins using a pigP protein trap library. Development. 141:39944005. doi:10.1242/dev.111054.

Mao, Y., and M. Freeman. 2009. Fasciclin 2, the Drosophila orthologue of neural cell-adhesion molecule, inhibits EGF receptor signalling. Development. 136:473481. doi:10.1242/dev.026054.

McNeill, H., M. Ozawa, R. Kemler, and W.J. Nelson. 1990. Novel function of the cell adhesion molecule uvomorulin as an inducer of cell surface polarity. Cell. 62:309-316.

Morais-de-Sá, E., V. Mirouse, and D. St Johnston. 2010. aPKC phosphorylation of Bazooka defines the apical/lateral border in Drosophila epithelial cells. Cell. 141:509-523. doi:10.1016/j.cell.2010.02.040.

Myster, S.H., R. Cavallo, C.T. Anderson, D.T. Fox, and M. Peifer. 2003. Drosophila p120catenin plays a supporting role in cell adhesion but is not an essential adherens junction component. J Cell Biol. 160:433-449. doi:10.1083/jcb.200211083.

Nagai-Tamai, Y., K. Mizuno, T. Hirose, A. Suzuki, and S. Ohno. 2002. Regulated protein-protein interaction between aPKC and PAR-3 plays an essential role in the polarization of epithelial cells. Genes Cells. 7:1161-71.

Nassar, N., G.R. Hoffman, D. Manor, J.C. Clardy, and R.A. Cerione. 1998. Structures of Cdc42 bound to the active and catalytically compromised forms of Cdc42GAP. Nature Structural Biology. 5:1047-1052. doi:10.1038/4156.

Neisch, A.L., E. Formstecher, and R.G. Fehon. 2013. Conundrum, an ARHGAP18 orthologue, regulates RhoA and proliferation through interactions with Moesin. Mol Biol Cell. 24:1420-1433. doi:10.1091/mbc.E12-11-0800.

Nejsum, L.N., and W.J. Nelson. 2007. A molecular mechanism directly linking Ecadherin adhesion to initiation of epithelial cell surface polarity. J Cell Biol. 178:323-335. doi:10.1083/jcb.200705094.

Newsome, T.P., B. Asling, and B.J. Dickson. 2000. Analysis of Drosophila photoreceptor axon guidance in eye-specific mosaics. Development. 127:851860. 
Padrick, S.B., and M.K. Rosen. 2010. Physical mechanisms of signal integration by WASP family proteins. Ann Rev Biochem. 79:707-735. doi:10.1146/annurev.biochem.77.060407.135452.

Peterson, F.C., R.R. Penkert, B.F. Volkman, and K.E. Prehoda. 2004. Cdc42 regulates the Par-6 PDZ domain through an allosteric CRIB-PDZ transition. Mol Cell. 13:665-676. doi:10.1016/S1097-2765(04)00086-3.

Pignoni, F., and S.L. Zipursky. 1997. Induction of Drosophila eye development by decapentaplegic. Development. 124:271-278.

Prakash, S., J.C. Caldwell, D.F. Eberl, and T.R. Clandinin. 2005. Drosophila Ncadherin mediates an attractive interaction between photoreceptor axons and their targets. Nat Neurosci. 8:443-450. doi:10.1038/nn1415.

Qiu, R.G., A. Abo, and G. Steven Martin. 2000. A human homolog of the C. elegans polarity determinant Par-6 links Rac and Cdc42 to PKCzeta signaling and cell transformation. Curr Biol. 10:697-707. doi:10.1016/s0960-9822(00)00535-2.

Rodriguez, J., F. Peglion, J. Martin, L. Hubatsch, J. Reich, N. Hirani, A.G. Gubieda, J. Roffey, A.R. Fernandes, D. St Johnston, J. Ahringer, and N.W. Goehring. 2017. aPKC cycles between functionally distinct PAR protein assemblies to drive cell polarity. Dev Cell. 42:400-415.e9. doi:10.1016/j.devcel.2017.07.007.

Rodriguez-Boulan, E., and I.G. Macara. 2014. Organization and execution of the epithelial polarity programme. Nat Rev Mol Cell Biol. 15:225-242. doi:10.1038/nrm3775.

Schindelin, J., I. Arganda-Carreras, E. Frise, V. Kaynig, M. Longair, T. Pietzsch, S. Preibisch, C. Rueden, S. Saalfeld, B. Schmid, J.-Y. Tinevez, D.J. White, V. Hartenstein, K. Eliceiri, P. Tomancak, and A. Cardona. 2012. Fiji: an open-source platform for biological-image analysis. Nat Methods. 9:676-682. doi:10.1038/nmeth.2019.

Shaner, N.C., G.G. Lambert, A. Chammas, Y. Ni, P.J. Cranfill, M.A. Baird, B.R. Sell, J.R. Allen, R.N. Day, M. Israelsson, M.W. Davidson, and J. Wang. 2013. A bright monomeric green fluorescent protein derived from Branchiostoma lanceolatum. Nat Methods. 10:407-409. doi:10.1038/nmeth.2413.

Shulman, J.M., R. Benton, and D. St Johnston. 2000. The Drosophila homolog of $C$. elegans PAR-1 organizes the oocyte cytoskeleton and directs oskar mRNA localization to the posterior pole. Cell. 101:377-388. doi:10.1016/s00928674(00)80848-x.

Sousa, S., D. Cabanes, C. Archambaud, F. Colland, E. Lemichez, M. Popoff, S. Boisson-Dupuis, E. Gouin, M. Lecuit, P. Legrain, and P. Cossart. 2005. ARHGAP10 is necessary for alpha-catenin recruitment at adherens junctions and for Listeria invasion. Nature Cell Biol. 7:954-960. doi:10.1038/ncb1308.

St Johnston, D., and B. Sanson. 2011. Epithelial polarity and morphogenesis. Curr Opin Cell Biol. 23:540-546. doi:10.1016/j.ceb.2011.07.005. 
St Johnston, D., and J. Ahringer. 2010. Cell polarity in eggs and epithelia: parallels and diversity. Cell. 141:757-774. doi:10.1016/j.cell.2010.05.011.

Sui, L., S. Alt, M. Weigert, N. Dye, S. Eaton, F. Jug, E.W. Myers, F. Jülicher, G. Salbreux, and C. Dahmann. 2018. Differential lateral and basal tension drive folding of Drosophila wing discs through two distinct mechanisms. Nat Commun. 9:4620. doi:10.1038/s41467-018-06497-3.

Suzuki, A., M. Hirata, K. Kamimura, R. Maniwa, T. Yamanaka, K. Mizuno, M. Kishikawa, H. Hirose, Y. Amano, N. Izumi, Y. Miwa, and S. Ohno. 2004. aPKC acts upstream of PAR-1b in both the establishment and maintenance of mammalian epithelial polarity. Curr Biol. 14:1425-1435.

Szafranski, P., and S. Goode. 2006. Basolateral junctions are sufficient to suppress epithelial invasion during Drosophila oogenesis. Dev Dyn. 236:364-373. doi:10.1002/dvdy.21020.

Takahashi, K., T. Matsuo, T. Katsube, R. Ueda, and D. Yamamoto. 1998. Direct binding between two PDZ domain proteins Canoe and ZO-1 and their roles in regulation of the jun N-terminal kinase pathway in Drosophila morphogenesis. Mech Dev. 78:97-111. doi:10.1016/s0925-4773(98)00151-8.

Tepass, U., C. Theres, and E. Knust. 1990. crumbs encodes an EGF-like protein expressed on apical membranes of Drosophila epithelial cells and required for organization of epithelia. Cell. 61:787-799.

Tepass, U., E. Gruszynski-DeFeo, T.A. Haag, L. Omatyar, T. Torok, and V. Hartenstein. 1996. shotgun encodes Drosophila E-cadherin and is preferentially required during cell rearrangement in the neurectoderm and other morphogenetically active epithelia. Genes Dev. 10:672-685. doi:10.1101/gad.10.6.672.

Thompson, B.J., F. Pichaud, and K. Röper. 2013. Sticking together the Crumbs - an unexpected function for an old friend. Nat Rev Mol Cell Biol. 14:307-314. doi:10.1038/nrm3568.

Tian, A.-G., and W.-M. Deng. 2008. Lgl and its phosphorylation by aPKC regulate oocyte polarity formation in Drosophila. Development. 135:463-471. doi:10.1242/dev.016253.

Trushko, A., I. Di Meglio, A. Merzouki, C. Blanch-Mercader, S. Abuhattum, J. Guck, K. Alessandri, P. Nassoy, K. Kruse, B. Chopard, and A. Roux. 2020. Buckling of an epithelium growing under spherical confinement. Dev Cell. doi:10.1016/j.devcel.2020.07.019.

Van Itallie, C.M., A.J. Tietgens, A. Aponte, K. Fredriksson, A.S. Fanning, M. Gucek, and J.M. Anderson. 2014. Biotin ligase tagging identifies proteins proximal to Ecadherin, including lipoma preferred partner, a regulator of epithelial cell-cell and cell-substrate adhesion. J Cell Sci. 127:885-895. doi:10.1242/jcs.140475. 
Waaijers, S., J.J. Ramalho, T. Koorman, E. Kruse, and M. Boxem. 2015. The $C$. elegans Crumbs family contains a CRB3 homolog and is not essential for viability. Biol Open. 4:276-284. doi:10.1242/bio.201410744.

Wang, A.Z., G.K. Ojakian, and W.J. Nelson. 1990. Steps in the morphogenesis of a polarized epithelium. I. Uncoupling the roles of cell-cell and cell-substratum contact in establishing plasma membrane polarity in multicellular epithelial (MDCK) cysts. J Cell Sci. 95 ( Pt 1):137-151.

Wang, Y.-C., Z. Khan, M. Kaschube, and E.F. Wieschaus. 2012. Differential positioning of adherens junctions is associated with initiation of epithelial folding. Nature. 484:390-393. doi:10.1038/nature10938.

Watabe, M., A. Nagafuchi, S. Tsukita, and M. Takeichi. 1994. Induction of polarized cell-cell association and retardation of growth by activation of the E-cadherincatenin adhesion system in a dispersed carcinoma line. J Cell Biol. 127:247-256. doi:10.1083/jcb.127.1.247.

Waterhouse, A., M. Bertoni, S. Bienert, G. Studer, G. Tauriello, R. Gumienny, F.T. Heer, T.A.P. de Beer, C. Rempfer, L. Bordoli, R. Lepore, and T. Schwede. 2018. SWISS-MODEL: homology modelling of protein structures and complexes. Nucleic Acids Res. 46:W296-W303. doi:10.1093/nar/gky427.

Wei, J., M. Hortsch, and S. Goode. 2004. Neuroglian stabilizes epithelial structure during Drosophila oogenesis. Dev Dyn. 230:800-808. doi:10.1002/dvdy.20108.

Whitney, D.S., F.C. Peterson, A.W. Kittell, J.M. Egner, K.E. Prehoda, and B.F. Volkman. 2016. Binding of Crumbs to the Par-6 CRIB-PDZ module is regulated by Cdc42. Biochemistry. 55:1455-1461. doi:10.1021/acs.biochem.5b01342.

Wirtz-Peitz, F., T. Nishimura, and J.A. Knoblich. 2008. Linking cell cycle to asymmetric division: Aurora-A phosphorylates the Par complex to regulate Numb localization. Cell. 135:161-173. doi:10.1016/j.cell.2008.07.049.

Yamanaka, T., Y. Horikoshi, A. Suzuki, Y. Sugiyama, K. Kitamura, R. Maniwa, Y. Nagai, A. Yamashita, T. Hirose, H. Ishikawa, and S. Ohno. 2001. PAR-6 regulates aPKC activity in a novel way and mediates cell-cell contact-induced formation of the epithelial junctional complex. Genes Cells. 6:721-731.

Zhao, Z., and E. Manser. 2015. Myotonic dystrophy kinase-related Cdc42-binding kinases (MRCK), the ROCK-like effectors of Cdc42 and Rac1. Small GTPases. 6:81-88. doi:10.1080/21541248.2014.1000699.

Zihni, C., E. Vlassaks, S. Terry, J. Carlton, T.K.C. Leung, M. Olson, F. Pichaud, M.S. Balda, and K. Matter. 2017. An apical MRCK-driven morphogenetic pathway controls epithelial polarity. Nat Cell Biol. 19:1049-1060. doi:10.1038/ncb3592. 
Table and Figure legends:

Table 1. List of Drosophila GAPs

Table 2. Drosophila GAPs ranked by the predicted strength of their interactions with Cdc42

Figure 1. Cdc42 is essential for the establishment of cell polarity in Drosophila follicle cells.

A) A stage 7 egg chamber containing multiple $c d c 42^{[2]}$ mutant follicle cell clones (marked by the loss of RFP, magenta) stained for Armadillo (white) and DAPI (blue) expressing endogenously-tagged GFP-aPKC (green). The mutant cells are marked by arrows. GFP-aPKC is lost from the apical side of $\mathrm{c} d c 42$ mutant follicle cells. In some cells, GFP-aPKC co-localises with Armadillo in puncta. Cells lacking Cdc42 become round and often lose contact with neighbouring cells, resulting in breaks in the epithelial layer. In other cases, the $c d c 42$ mutant cells lie basally to to wild-type cells. Scale bars $10 \mu \mathrm{m}$.

B) A diagram showing the interface between $\mathrm{Cdc} 42$ and bound

ARHGAP1/RhoGAP19D. Key amino acids that mediate the interaction are shown in purple for $\mathrm{Cdc} 42$ and in green for ARHGAP1. Amino acid changes that are predicted to strengthen the interaction between Cdc42 and RhoGAP19D are shown in parentheses.

C) Alignment of Drosophila GAPs and human ARHGAP1. Conserved amino acids involved in the interaction with $\mathrm{Cdc} 42$ are indicated by black arrows. The green arrows mark the variable amino acids that are predicted to strengthen the interaction between Cdc42 and RhoGAP19D. 
Figure 2. RhoGAP19D is required for the integrity of epithelial layer.

A) Comparison of the domain structure of Drosophila RhoGAP19D with its orthologues, human ARHGAP23/21 and C. elegans PAC-1. RHDOGAP19D contains PDZ and GAP domains, but lacks a PH domain.

B) Diagram showing the CRISPR-induced mutations in RhoGAP19D. The mutations generate proteins that lack the GAP domain, but still contain the PDZ domain.

C) A stage 7 rhogp19d mutant egg chamber showing invasions of the follicle cell epithelium into the adjacent germ line. The mutant cells (white arrows) invade between the nurse cells at the anterior of the egg chamber. Phalloidin staining is shown in green and DAPI in blue.

D) Graph showing the number of wild-type and rhogap19d mutant cells per egg chamber at stage 8 .

E) Invading rhogap 19d mutant cells (marked by the loss of RFP, magenta) maintain their adherens junctions and epithelial organisation, as shown by E-cadherin-GFP expression; DAPI (blue).

F) PH3 staining (green) of mitotic cells in a stage 5 egg chamber containing a rhogap19d mutant clone (marked by the loss of RFP, magenta). There is no increase in cell division in the mutant clone.

G) Quantification of the number of mitoses in wild-type and rhogap19d mutant egg chambers during stages 4-5.

H) mNeonGreen-RHOGAP19D (green)localises to the lateral domain of the follicle cells and is slightly enriched at the adherens junctions stained with Cno (white); DAPI (blue). RHOGAP19D protein in the germline was depleted by RNAi.

I) Surface view of wild-type cells expressing mNeonGreen-RhoGAP19D 
J) and K) mNeonGreen-RHOGAP19D localises normally in p120 catenin $^{308} /+$ and p120 catenin $^{308}$ homozygous cells.

$\mathrm{L}$ and $\mathrm{M}$ ) mNeonGreen-RHOGAP19D recruitment to lateral domain is almost lost in cells expressing $\alpha$-catenin RNAi (L) and is strongly reduced in shotgun $^{\mathrm{IG} 29}$ mutant clones (marked by the loss of RFP, magenta, M).

N) mNeonGreen-RHOGAP19D localisation is not affected in $\lg l^{4}$ mutant clones (marked by the loss of RFP, magenta) compared to wild-type cells. Scale bars $10 \mu \mathrm{m}$.

\section{Figure 3. RHOGAP19D inhibits CDC42 activity laterally.}

A) The CDC42 effector, N-WASP (tagged with mNeonGreen) spreads laterally in rhogap19D mutant cells (marked by the loss of RFP, magenta).

B) Cartoon showing the UAS- GrabFP-A-int system.

C) mCherry-Grab FP Bazooka predominantly localizes to apical side of the follicle cells when expressed under the control of TJ-Gal4, whereas GFP-RhoGAP19D alone localizes laterally.

D) Co-expression of mCherry-Grab FP Baz and GFP-RhoGAP19D results in the apical recruitment of RhoGAP19D, leading to a loss of epithelial and mis-localisation of aPKC (white). Scale bars $10 \mu \mathrm{m}$.

\section{Figure 4. rhogap19D mutant cells are taller and have an enlarged apical}

\section{domain.}

A-E) Regions of the stage 7 follicle cell epithelium stained with DAPI (blue) containing clones of rhogap19d mutant cells marked by the loss of RFP (magenta). 
(A) Cell mask staining (green) of the plasma membranes reveals that mutant cells are taller than wild-type cells and have domed apical surfaces. The arrows indicate the apical surfaces of the mutant cells.

B) The adherens junctions marked by endogenously-tagged E-Cad-GFP (green) form at the same level in rhogap19d mutant and wild-type cells. (Phalloidin; white).

C) In rhogap19d mutant cells, GFP-aPKC localises all around the apical domain above the adherens junctions (marked by Cno staining; white).

D) Crb-GFP marks an enlarged sub-apical region in rhogap19d cells.

E) rhogap19d mutant cells have slightly shorter lateral domains than wild-type cells, as shown by Lgl-GFP localisation (green).

F) A graph showing the mean cell height in wild-type and rhogap19d mutant cells.

G) A graph showing the relative position of adherens junctions compared to total cell height in wild-type and rhogap19d mutant cells.

H) A graph showing the mean apical domain length in wild-type and rhogap19d mutant cells.

I) A graph showing the mean lateral domain length in wild-type and rhogap19d mutant cells. Scale bars $10 \mu \mathrm{m}$.

\section{Figure 5. rhogap19d mutant cells are more motile and contract laterally.}

A) Single frames and a temporal projection of a time-lapse movie of a stage 7 egg chamber containing a large rhogap19d mutant clone (marked by the loss of RFP; magenta) and expressing GFP-aPKC (green). The blurred apical surfaces of the mutant cells in the temporal projection indicate that they are moving between frames 
B) Magnification of the boxed areas in (A), showing that rhogap19d mutant cells

(bottom panels) are more motile than wild-type cells (top panels). Temporal

projections after 1, 2 and 4 mins.

C) A single frame from a movie showing a cluster of rhogap19d mutant cells (marked by the loss of RFP, magenta) beginning to invade the germ line. The cells in the cluster appear to have detached from the basement membrane. (Cell mask; white). The lower panel shows a magnification of the boxed area.

D) rhogap19d mutant cells have lateral foci of non-muscle Myosin II foci (ZipperGFP; green) and reduced levels at the apical side, compared to wild-type cells. (DAPI; blue).

E) Gek (green) localises apically in wild-type follicle cells, but extends along the lateral domain of rhogap19d mutant cells. DAPI (blue). Scale bars $10 \mu \mathrm{m}$.

\section{Figure 6. Genetic interactions between rhogap19d and other polarity factors.}

A histogram showing the penetrance of the germline invasion phenotype of large rhogap 19d mutant clones in combination with other polarity mutants. Removing one copy of scrib, $\operatorname{lgl}$ or Pakl strongly enhances the penetrance of the invasion phenotype. rhogap19d fasII double mutant clones and rhogap19d clones in which $n r g$ has been depleted by RNAi also show a highly penetrant invasive phenotype. Loss of one copy of $a P K C$, gek, crb or par- 1 strongly reduces the frequency of invasions.

\section{Figure 7. A revised network of inhibitory interactions between polarity factors}

a) A diagram showing how the recruitment of RhoGAP19D to the lateral membrane by E-cadherin adhesion complexes restricts Cdc42 activity to the apical domain. This 
adds a new inhibitory interaction to the network of interactions between apical and lateral polarity factors.

b) A model of the changes in rhogap19d mutant cells that lead to the invasive phenotype. In the absence of RhoGAP19D, active Cdc42 localises along the lateral, as well as the apical domain, and activates Gek to induce lateral actomyosin contractions. Lateral Cdc42-GTP also alters the conformation of Par-6/aPKC so that it is competent to bind to Crumbs. This primed Par-6/aPKC then diffuses until it binds to apical Crumbs, which activates aPKC's kinase activity, resulting in expansion of the apical domain and a dome-shaped apical surface. 


\section{Table 1}

\begin{tabular}{|c|c|c|c|}
\hline Gene symbol & Gene name & Other names & UniProt \\
\hline CdGAPr & protein-related & GAP, d-CdGAPr & Q9VIS1 \\
\hline conu & conundrum & & Q8T0G4 \\
\hline $\mathrm{CV}-\mathrm{c}$ & crossveinless c & RhoGAP88C & A8JR05 \\
\hline Graf & GRAF ortholog (h. sapiens) & & X2JDY8 \\
\hline Ocrl & syndrome of Lowe & EG:86E4.5 & Q95R90 \\
\hline RacGAP84C & protein at $84 \mathrm{C}$ & rnRacGAP & P40809 \\
\hline RhoGAP1A & protein at $1 \mathrm{~A}$ & EG:23E12.2 & Q6W436 \\
\hline RhoGAP5A & protein at $5 \mathrm{~A}$ & & Q9W4A9 \\
\hline RhoGAP15B & protein at $15 B$ & & Q0KHR5 \\
\hline RhoGAP16F & protein at $16 \mathrm{~F}$ & & Q9VWY8 \\
\hline RhoGAP18B & protein at $18 \mathrm{~B}$ & whir & Q9VWL7 \\
\hline RhoGAP19D & protein at $19 \mathrm{D}$ & & Q9VRA6 \\
\hline RhoGAP54D & protein at $54 \mathrm{D}$ & & A1ZAW3 \\
\hline RhoGAP68F & protein at $68 \mathrm{~F}$ & CG 6811 & M9PC96 \\
\hline RhoGAP71E & protein at $71 \mathrm{E}$ & I(3)j6B9 & B7Z058 \\
\hline RhoGAP92B & protein at 92B & & A0A0B4LHC1 \\
\hline RhoGAP93B & protein at 93B & CrGAP & Q9VDE9 \\
\hline RhoGAP100F & protein at $100 \mathrm{~F}$ & Syd-1 & Q9V9S7 \\
\hline RhoGAP102A & protein at $102 \mathrm{~A}$ & Dm_4:1183 & H9XVN1 \\
\hline RhoGAPp190 & protein at 190 & p190RhoGAP, p190 RhoGAP & Q9VX32 \\
\hline Rlip & Ral interacting protein & dRalBP, D-RLIP & Q9VDG2 \\
\hline tum & tumbleweed & racGAP50C, acGAP, RacGAP, DRacGAP & Q9N9Z9 \\
\hline
\end{tabular}

Table 1. List of GAPs in Drosophila.

The table shows all known GAPs with their additional names and UniProt numbers. 


\section{Table 2}

\begin{tabular}{|c|c|c|c|c|c|c|c|}
\hline Protein 1 & Gene 1 & \%id1 & Protein 2 & Gene 2 & \%id2 & PDB & Z-score \\
\hline Q9VRA6-RhoGAP & RhoGAP19D & 24 & P40793-RAS & Cdc42 & 94 & 1AM4 & 3.329 \\
\hline M9PC96-RhoGAP & RhoGAP68F & 40 & P40793-RAS & Cdc42 & 92 & $1 \mathrm{GRN}$ & 3.122 \\
\hline Q9VIS1-RhoGAP & CdGAPr & 26 & P40793-RAS & Cdc42 & 92 & $1 \mathrm{GRN}$ & 2.999 \\
\hline A0A0B4LHC1-RhoGAP & RhoGAP92B & 36 & P40793-RAS & Cdc42 & 92 & 2NGR & 2.9 \\
\hline P40809-RhoGAP & RacGAP84C & 26 & P40793-RAS & Cdc42 & 92 & $1 \mathrm{GRN}$ & 2.88 \\
\hline Q8T0G4-RhoGAP & conu & 26 & P40793-RAS & Cdc42 & 92 & $1 \mathrm{GRN}$ & 2.835 \\
\hline Q9VDE9-RhoGAP & RhoGAP93B & 30 & P40793-RAS & Cdc42 & 92 & $1 \mathrm{GRN}$ & 2.823 \\
\hline X2JDY8-RhoGAP & Graf & 29 & P40793-RAS & Cdc42 & 94 & $1 \mathrm{AM} 4$ & 2.748 \\
\hline Q0KHR5-RhoGAP & RhoGAP15B & 28 & P40793-RAS & Cdc42 & 94 & $1 \mathrm{AM} 4$ & 2.723 \\
\hline A8JR05-RhoGAP & $\mathrm{CV}-\mathrm{C}$ & 29 & P40793-RAS & Cdc42 & 94 & 1AM4 & 2.642 \\
\hline Q9VWL7-RhoGAP & RhoGAP18B & 28 & P40793-RAS & Cdc42 & 94 & $1 \mathrm{AM} 4$ & 2.488 \\
\hline Q9VWY8-RhoGAP & RhoGAP16F & 22 & P40793-RAS & Cdc42 & 94 & 1AM4 & 2.142 \\
\hline Q9N9Z9-RhoGAP & tum & 22 & P40793-RAS & Cdc42 & 94 & $1 \mathrm{AM} 4$ & 1.951 \\
\hline Q9VX32-RhoGAP & RhoGAPp190 & 28 & P40793-RAS & Cdc42 & 92 & 1GRN & 1.93 \\
\hline Q9VDG2-RhoGAP & Rlip & 32 & P40793-RAS & Cdc42 & 92 & 2NGR & 1.683 \\
\hline
\end{tabular}

GAPs ranked by the predicted strength of their interaction with $\mathrm{Cdc} 42$. RhoGAP19D has the highest Z-score. 
bioRxiv preprint doi: https://doi.org/10.1101/2020.09.17.300145; this version posted September 21, 2020. The copyright holder for this

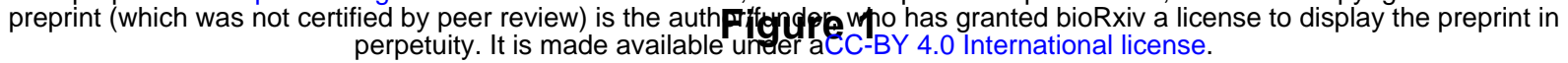

A
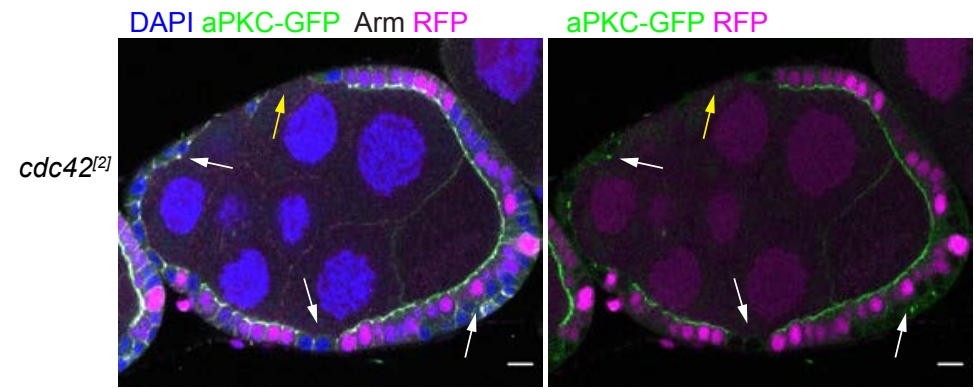

Arm RFP
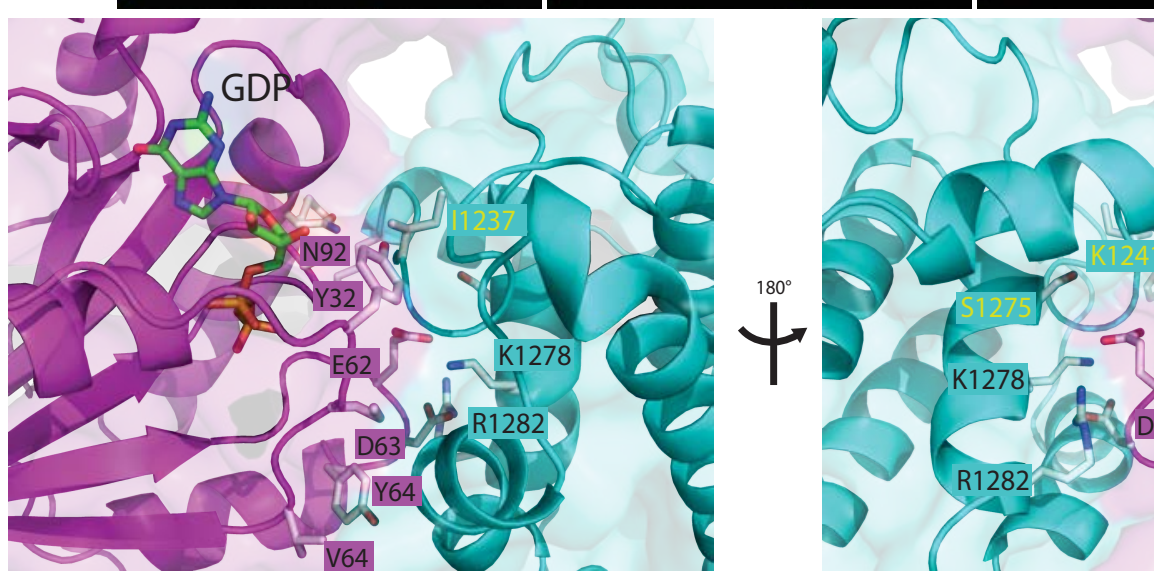

C

Ile1237 Lys1241

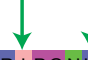

RhoGAP19D CPMSKVNAYVPHLVEVCTNIVE TKGLGVV

$\begin{array}{lll}1 \text { IIn:B } & \text { LQEKNPEQEPIPIVLRETVAYLQ } & \text { AHALTTE } \\ \text { CdGAPr } & \text { EHLLNSGQDIPMVLRCAEFIE NYGVID }\end{array}$

$\begin{array}{lll}\text { CdGAPr } & \text { EHLLNSGQDIPMVLRSCAEFIE } & \text { NYGVID } \\ \text { Graf } & \text { EAYHLDEAGFMFIRRCIQVLE IRGLED }\end{array}$

$\begin{array}{lll}\text { Graf } & \text { EAYHLDEAGFMF IRRCIQVLE } & \text { IRGLEDE } \\ \text { RhOGAP5A MVQLEPHHQIPFVVRRCVEEVE } & \text { ARGMLQE }\end{array}$

RhoGAP92B HLTSTNRE ISYIVELCCCCLL EHGLEEE

RhOGAP68F VMNSPCLNSIPPIVRKCVDSLSITGVIDTE

RhoGAP18B ELNMTDHPNVPRFVVDVCAYIEQPECIEQD

RhOGAP15B GDQQLTRYDVPVIVDKCINFVY I HGSMSE

RhOGAP100F SKNAPGSAPVPIVLRRCVEEVE RRGLDII

RhOGAP102A AYQEHSQLMVPIFVNNCIDYLE DNGLQQV

RhoGAP102A AYQEHSQLMVP IFVNNC IDYLE DNGLQQV
tum DFAPSIAPMIPAL IVHCVNEIE ARGLTEV

lum
RhoGAPp190 LMITSIAPM I PAL I VHCVNE IE ARGLITEV

RaCGAP84C DYAPRVAPMVPALIVHCVTEIE ARGLQQE

CV-C RhoGAP16F

RhoGAP93B

Rlip

MILQRSGQTLPLAVRAAFRWLQ LNALDQV
VLKHDQHRTVPRIVVDCCDLIEQKYRRSTQPI

con

QKEKFPFRKLPWIQTTLSEHVLLLNGKQTE
RSRCHDGVDIPLVVRDCIDFLQ DHLKCE

RSRCHDGVDIPLVVRDCIDFLQ DHLKC
QVTGTDSSLVPLFLEKL IGELL RRGSRE
CKHNNN IPGPLLVLILKLN KESPNR CKHNNNIPGPLLVLI ILKLN KESPNRR
DPGFLMHLQELKEFLMLEKNLTQE

GIYRIPGNKAAISELSEL VNTKDFQFESCAS

GIFRRSANTQVVREVQQK YNMGLPVDFDQYN

GIYRLSG ITSN IQRLRRA FDEERVPDLGNPE
GIYRKSGVGTK I SKLLALGLNQKESDDVFVDD

GI YRKSGVGTK I SKLL LALL NQKESDDVFVDD
GIYRVSGFADE IEALKLA LDREGEKTDMSET GLLRVGCASTKLRRMKHA LEAQHVKTPLPLD GIFRRSGNHSEIMALKER VNRGEDVDLKSVN GLYRASGNKVLVDELRKK LTHVYDPRWLKTD GI YRKSGSENSMHKLMSA FRADAFNVE I TRN GLYRLCGSATKKRLLREA FERNSRAVELTPE GLFRVSTSKKRVKQLREE FDKDIYFGISVDT GLYRLSSSEREYKALKEQ FLRGKATPHLGNT GIYRVPGSRAHVDMLFQR FEEDTNTEIDALD GLYRVSSTREKCKRLRRK LLRGKSTPHLGNK GIFRKSGGKSRIMKLREQ IEVTDSTAECMDV I YRQCGDYNK I QTLRFS I I ANDYDSLRQPD
IFRVSADVDEVGMKNR LDRWDPDYKNTL GIFRVSADVDEVGCMKNR LDRWDVPDYKNTL GLLRIGGHKQKTELLYNE
DVFRAPGHQGAMKKL I HF GLFRKAGAVSRQNELRMH

LESTFYQNPDNLD
LQAGRLVNVDNYS
IQHDKPLNLELAG

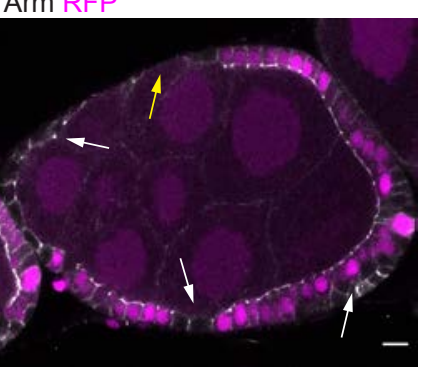

RhoGAP54

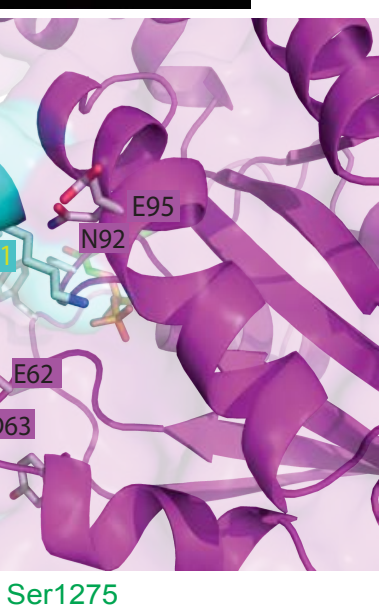

$\downarrow \stackrel{\text { Lys } 1278}{\downarrow} \downarrow^{\downarrow} 1282$

DRWEDVNVVSSLLKLF IRSLPDALMPASYY INF IE
EL HLPAVILKTFLRELPEPLLTFDLYPHVVGF MKQDI HAVSSLLKMYFRELPNPLCTYQLYDNFVEA RDLMESNT I ASAL KMYLRNL NEPLMTYQYHSDF IEA AYGNVNV IAGTLKLYLRLLPVPL I TFQAYPSFMAA YQDPHVIGSILKLYLRELPEPLLTYNLYKDFIRI VHVIAGLLKSFLRDLAEPLLTFELYEDVTGF DI HTLTSLHKQFFRELTSPLITQEAYERL.

EYNEHDVANVLKRFMRDLPERLLG KLTDSFVFV HVPDINVITGVLKDYLRELPEPLFTRCLFOMTVDA CPHDVATLLKEFLRDLPEPLLCNTLYLTFLKT CPHDVATLLKEF D IYVLCCCVKDFLRSLTEPL IPTSQWKDFANA
IPVNAVATALKDFFSKRLPPLFSKDIIKELEE IA DTHTLCCCVKDFLRQLVHPLIPIYHRRDFEEA FDLQQAYDVADMLKQYFRELPESLLTTKMSETFVA I LQQAYVADMLKQYFRELPESLLTTKMSETFVAI
VDIHTLTGVLKLFREIKSPLVRVNEAKTFIIGQ VDAHTPASLLKLWYRELYDPL I PDAYYEDCVNT VDAHTPASLLKLWYRELYDPL IPDAYYEDCVNT
NLPTACSLLKLFLRELPEPLLTTDLVARFEEV ATVHELSSLLKRWLRELPQPLLTNEL IQLFYQC FSAHDCATVFKGFLSELPEPLLTDAHYPAHLQ IA Met1346 Ile1353 Pro1357

RhoGAP19D 1 grn:B

CdGAPr

Graf

RhoGAP5A

RhoGAP92B

RhoGAP68F

RhoGAP18B

RhoGAP15B

RhoGAP100F

RhoGAP102A

tum

RhoGAPp190

RacGAP84C

CV-C

RhoGAP16F

RhoGAP93B

Rlip

conu

RhoGAP71E

RhoGAP54D

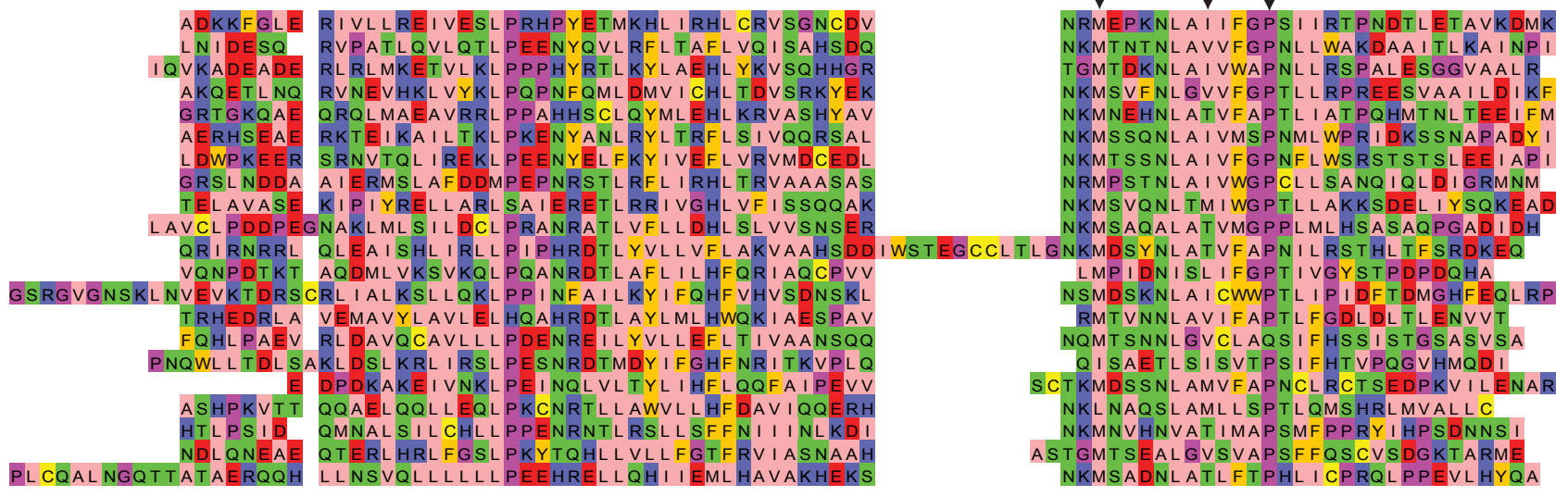

Figure 1.

Cdc42 is essential for the establishment of cell polarity in Drosophila follicle cells.

A) A stage 7 egg chamber containing multiple cdc42[2] mutant follicle cell clones (marked by the loss of RFP, magenta) stained for Armadillo (white) and DAPI (blue) expressing endogenously-tagged GFP-aPKC (green). Mutant cells are marked by white arrows. GFP-aPKC is lost from the apical side of cdc42 mutant follicle cells. In some cells, GFP-aPKC co-localises with Armadillo in puncta. Cells lacking Cdc42 become round and often lose contact with neighbouring cells, resulting in breaks in the epithelial layer (yellow arrow). In other cases, the cdc42 mutant cells lie basally to wild-type cells. Scale bars $10 \mu \mathrm{m}$.

B) A diagram showing the interface between Cdc42 and bound ARHGAP1/RhoGAP19D. Key amino acids that mediate the interaction are shown in purple for Cdc42 and in green for ARHGAP1. Amino acid changes that are predicted to strengthen the interaction between Cdc42 and RhoGAP19D are shown in yellow.

C) Alignment of Drosophila GAPs and human ARHGAP1. Conserved amino acids involved in the interaction with Cdc42 are indicated

by black arrows. The green arrows mark the variable amino acids that are predicted to strengthen the interaction between Cdc42 and RhoGAP19D. 
bioRxiv preprint doi: https://doi.org/10.1101/2020.09.17.300145; this version posted September 21, 2020. The copyright holder for this

preprint (which was not certified by peer review) is the author/funder, who has granted bioRxiv a license to display the preprint in perpetuity. It is made available under aCC-BY 4.0 International license.

Figure 2

A
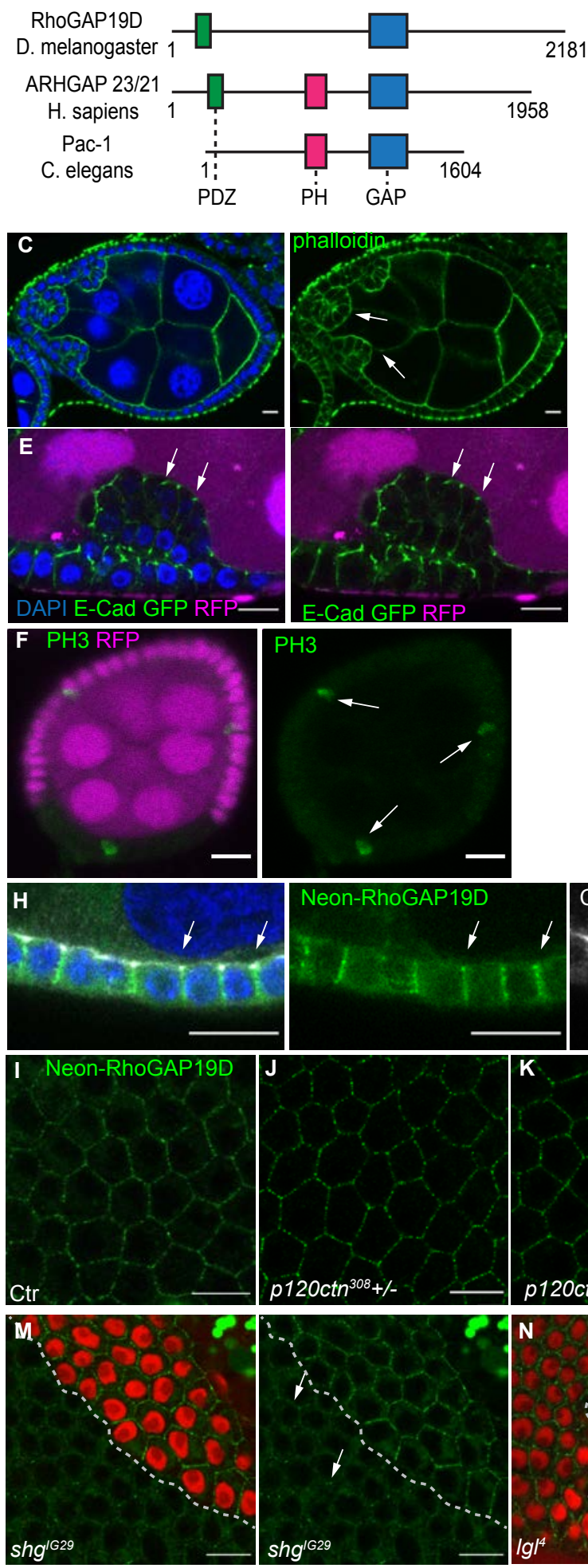

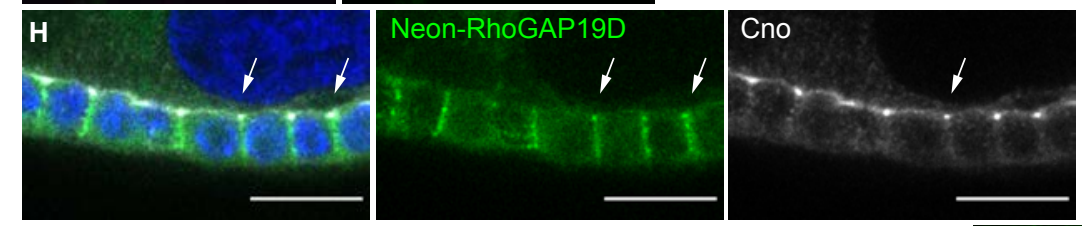

B

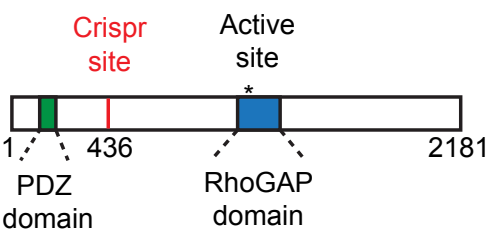

D

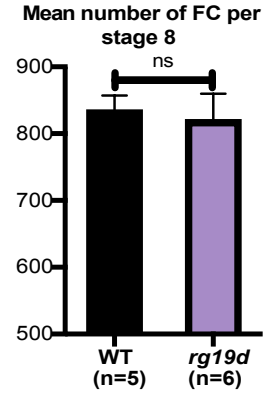

G

Mean number of mitosis

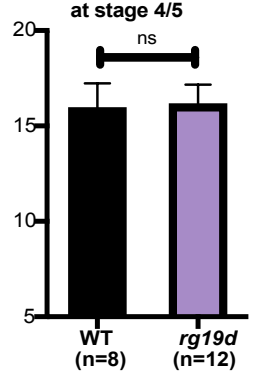

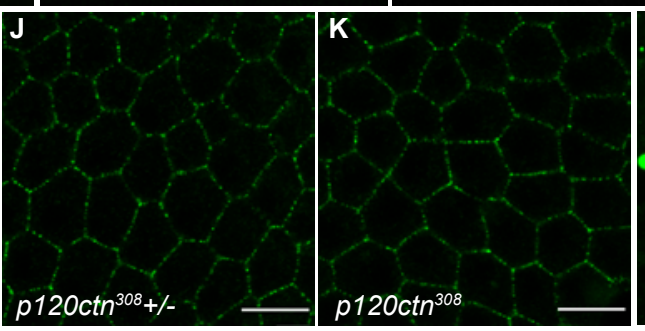
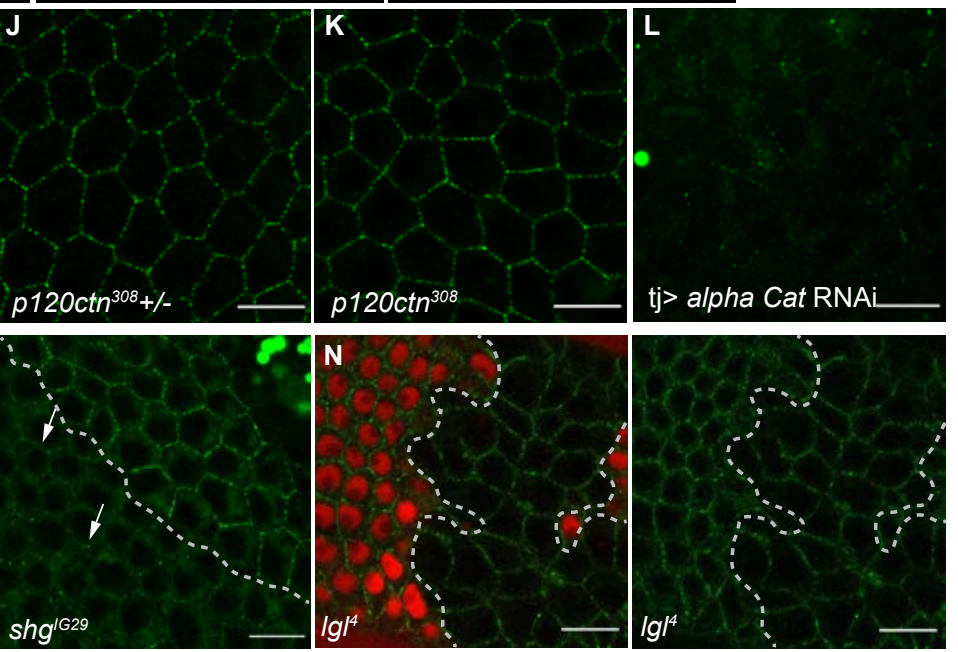
bioRxiv preprint doi: https://doi.org/10.1101/2020.09.17.300145; this version posted September 21, 2020. The copyright holder for this preprint (which was not certified by peer review) is the author/funder, who has granted bioRxiv a license to display the preprint in perpetuity. It is made available under aCC-BY 4.0 International license.

\section{Figure 3}

A

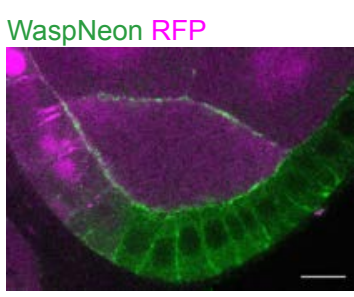

B

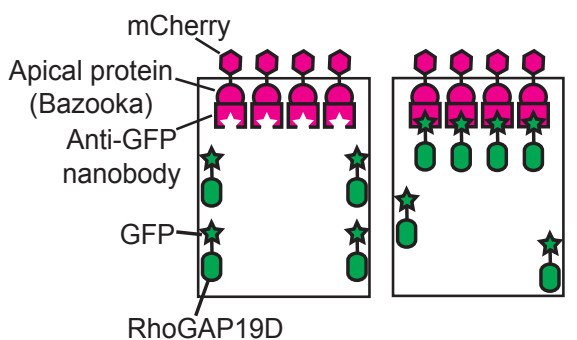

C

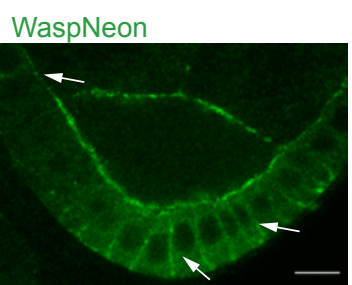

$\mathrm{tj}>\mathrm{m}$

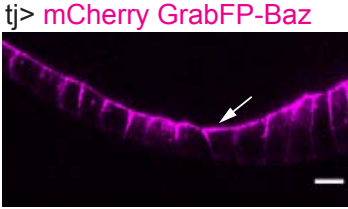

tj> GFP RhoGAP19D

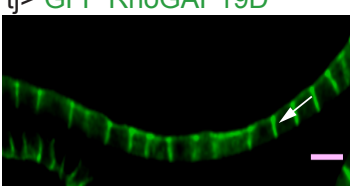

aPKC

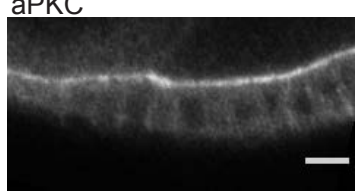

D tj> mCherry GrabFP-Baz + GFP RhoGAP19D
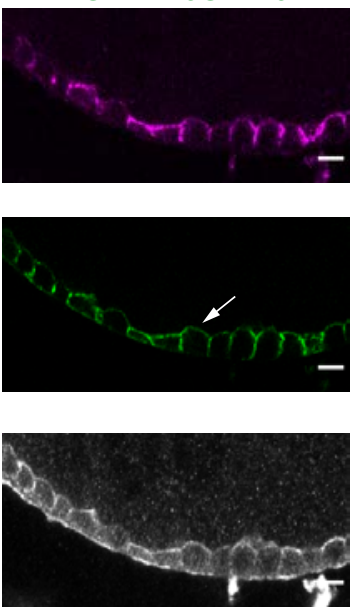
Figure 4
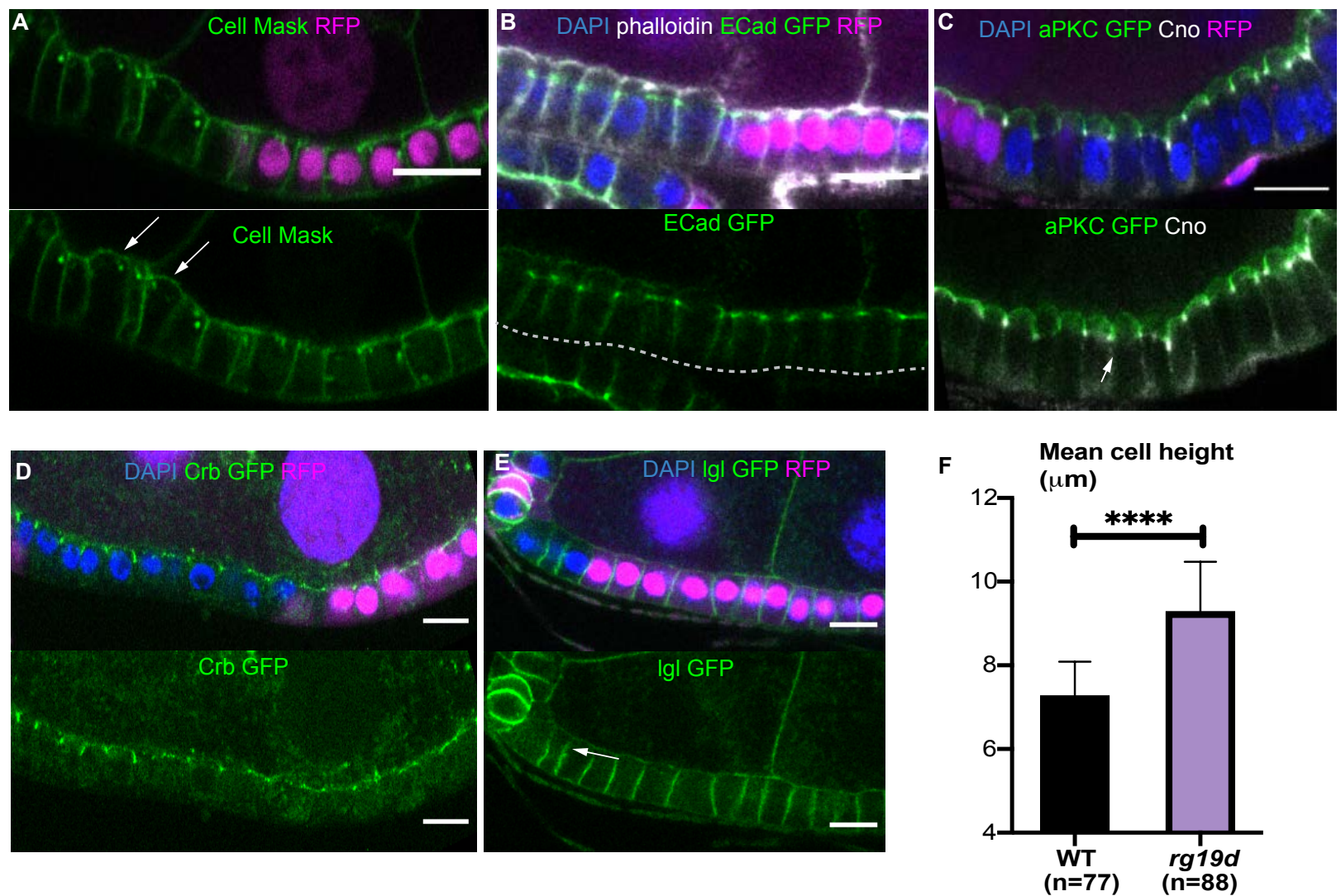

G

Relative position of junctions

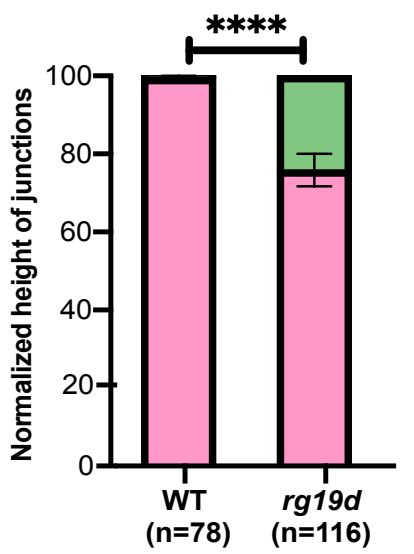

H

Mean apical domain length $(\mu \mathrm{m})$

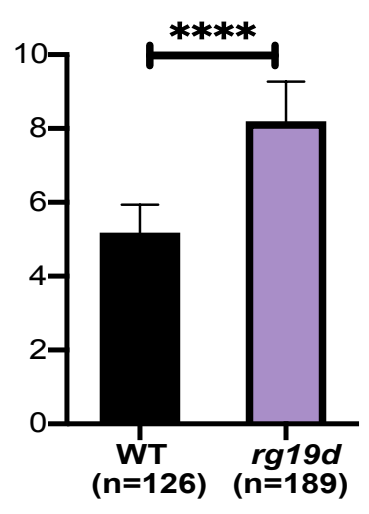

I

Mean lateral domain length $(\mu \mathrm{m})$

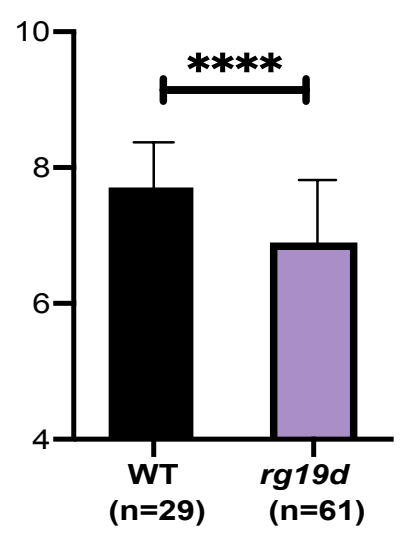


bioRxiv preprint doi: https://doi.org/10.1101/2020.09.17.300145; this version posted September 21, 2020. The copyright holder for this

preprint (which was not certified by peer review) is the author/funder, who has granted bioRxiv a license to display the preprint in perpetuity. It is made available under aCC-BY 4.0 International license.

Figure 5

A

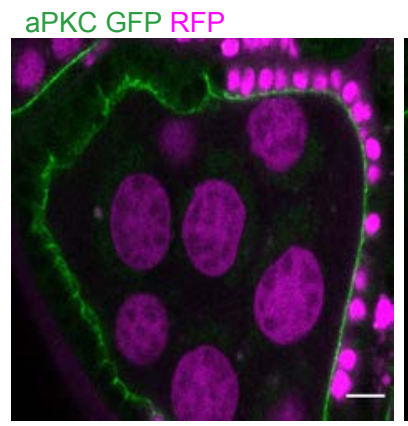

B

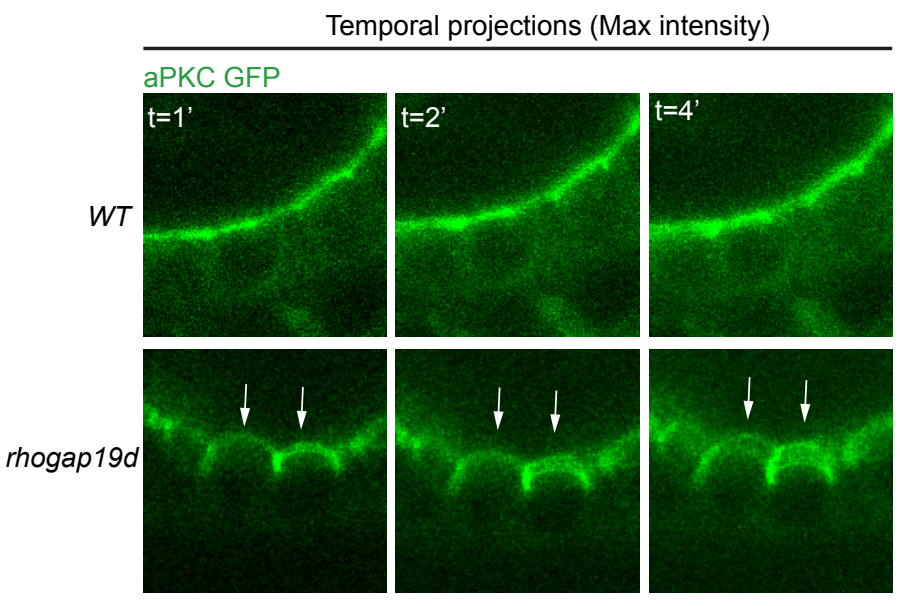

D

DAPI Zipper GFP RFP

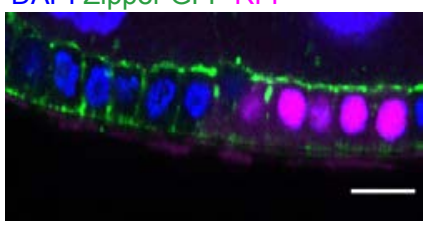

E

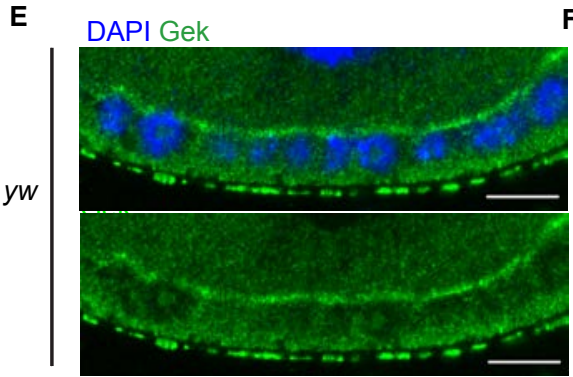

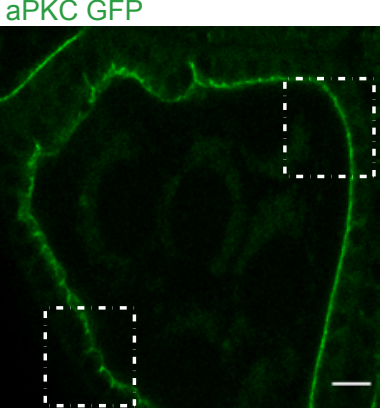

aPKC GFP

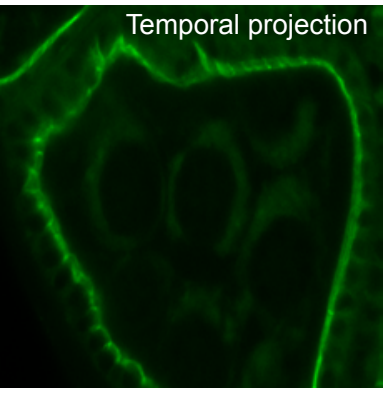

C Cell Mask RFP

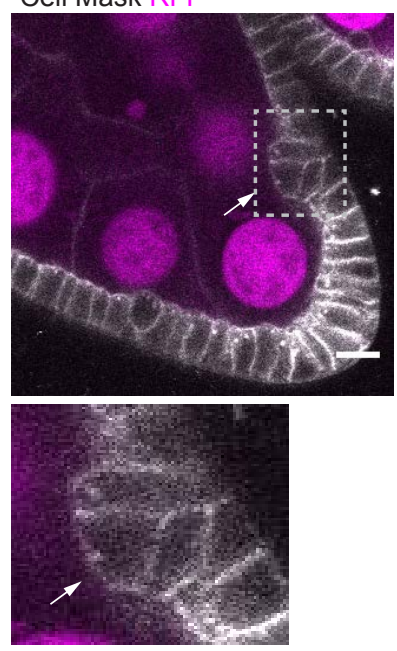

Zipper GFP
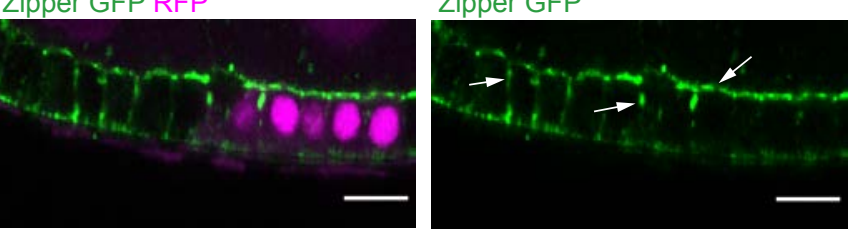

F $_{\text {DAPI Gek }}$

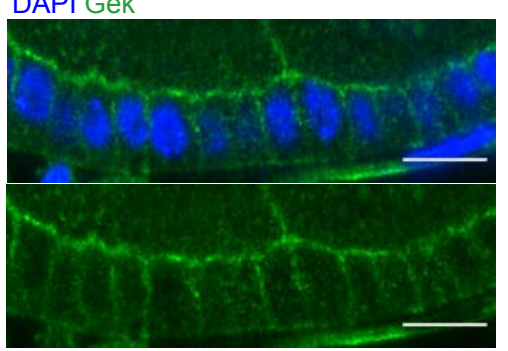


Fig. 6

Genetic interactions between rhogap19d and other apical/ lateral factors

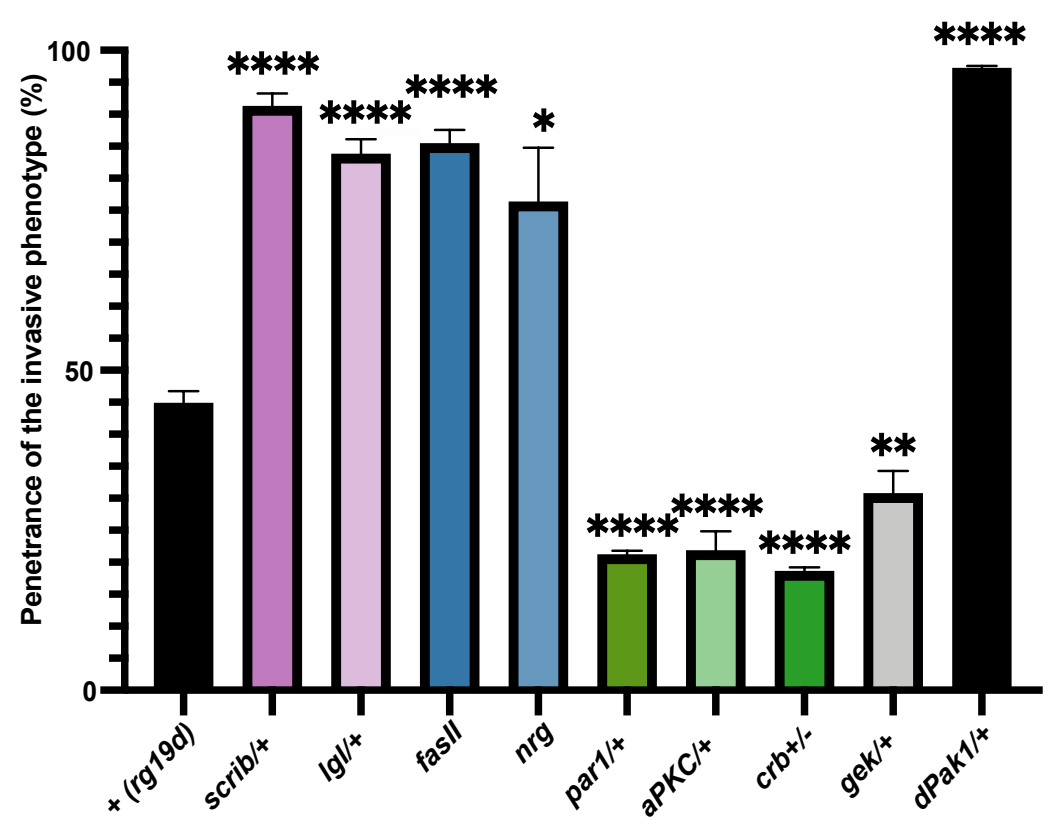


Figure 7

A

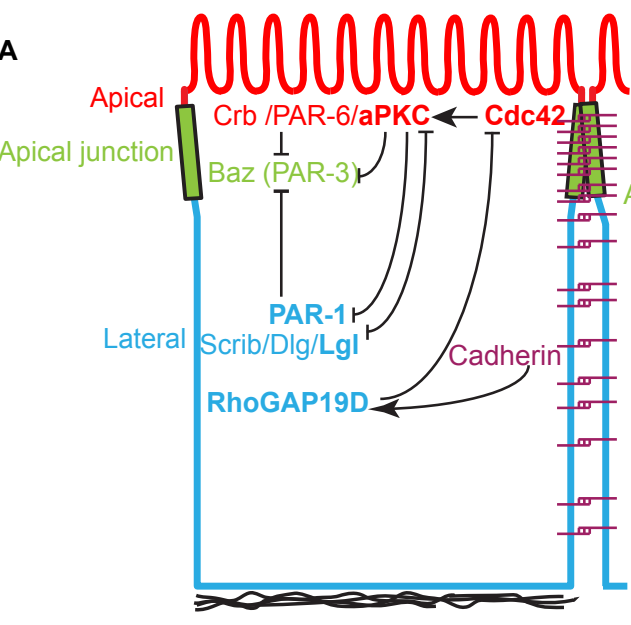

WT

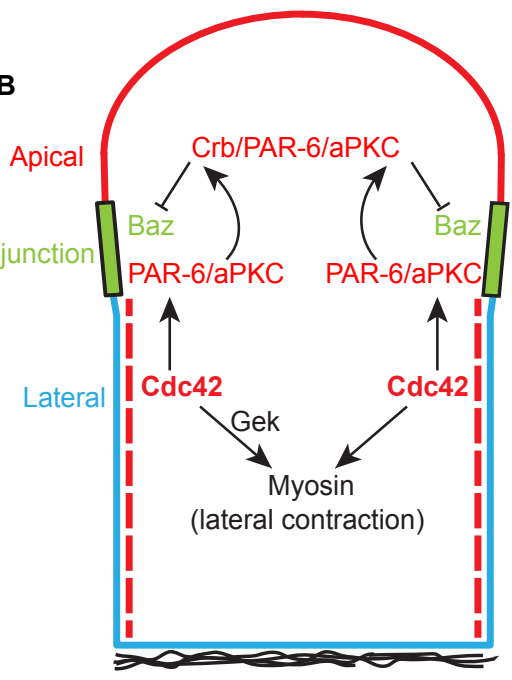

rg19d mutant 